\title{
Reformulations in mathematical programming: Automatic symmetry detection and exploitation
}

\author{
Leo Liberti
}

March 7, 2010

\begin{abstract}
If a mathematical program has many symmetric optima, solving it via Branchand-Bound techniques often yields search trees of disproportionate sizes; thus, finding and exploiting symmetries is an important task. We propose a method for automatically finding the formulation group of any given Mixed-Integer Nonlinear Program, and for reformulating the problem by means of static symmetry breaking constraints. The reformulated problem - which is likely to have fewer symmetric optima - can then be solved via standard Branch-and-Bound codes such as CPLEX (for linear programs) and CoUENNE (for nonlinear programs). Our computational results include formulation group tables for the MIPLib3, MIPLib2003, GlobalLib and MINLPLib instance libraries and solution tables for some instances in the aforementioned libraries.
\end{abstract}

Keywords group $\cdot$ symmetry $\cdot$ mixed integer nonlinear programming $\cdot$ branch and bound

\section{Introduction}

We consider Mixed-Integer Nonlinear Programs (MINLPs) in the following general form:

$$
\left.\begin{array}{rl}
\min _{x \in \mathbb{R}^{n}} f(x) & \\
g(x) & \leq b \\
x & \in\left[x^{L}, x^{U}\right] \\
\forall i \in Z \quad x_{i} & \in \mathbb{Z},
\end{array}\right\}
$$

where $f: \mathbb{R}^{n} \rightarrow \mathbb{R}, g: \mathbb{R}^{n} \rightarrow \mathbb{R}^{m}, b \in \mathbb{R}^{m}, x^{L}, x^{U} \in \mathbb{R}^{n}$ and $Z \subseteq\{1, \ldots, n\}$. Throughout the paper, elements of groups are represented by means of permutations of either the column or the row space; permutations on the row space are denoted by left multiplication, and permutations on the column space by right multiplication. For a mathematical program $P$ we let $\mathscr{F}(P)$ be its feasible region and $\mathscr{G}(P)$ be the set of its global optima. For $x \in \mathbb{R}^{n}$ and $B \subseteq\{1, \ldots, n\}$, we let $x[B]=\left(x_{j} \mid j \in B\right)$ be the partial vector of $x$ restricted to the components in $B$. If $X \subseteq \mathbb{R}^{n}$, then $X[B]=\left\{x[B] \in \mathbb{R}^{|B|} \mid x \in X\right\}$.

Leo Liberti

LIX, École Polytechnique, 91128 Palaiseau, France

E-mail: liberti@lix.polytechnique.fr 
Problems (1), be they linear or nonlinear, may be solved either heuristically or exactly. The most widely used technique for solving (1) exactly is the Branch-and-Bound (BB) algorithm. BB is a tree-based search in the variable space where each node represents a subproblem of (1) whose feasible region is a subset of the feasible region of (1). A node is pruned when one of the following holds: (a) a global optimum for the node was found; (b) the node was proved to be infeasible; (c) a lower bound for the problem at the node has higher value than the value of the objective function evaluated at the current best solution (the incumbent). In all other cases, the node is branched into two or more subnodes the union of whose feasible regions is the same as the feasible region of the parent node. For Mixed-Integer Linear Programs (MILPs), branching occurs on the integer variables only, and BB terminates finitely [54]. Finite termination also occurs with some nonlinear problems [1,9], although in general BB applied to MINLPs — called spatial BB (sBB) - can only terminate finitely with an $\varepsilon$-approximate optimum for a given $\varepsilon>0$.

BB usually converges slowly on problems (1) whose solution set has many symmetries because many leaf nodes in the BB tree may contain (symmetric) global optima: hence, no node in the paths leading from the root to these leaf nodes can ever be pruned. So, in general, we expect symmetric problems to yield larger BB trees. It is worth pointing out, however, that we carried out a few experiments using other solution methods than BB: these provided evidence to the effect that local-search based heuristics usually find optima faster if there are many of them - so it may not be worth breaking symmetries when using a heuristic method.

In this paper we describe methods to speed up the BB solution process applied to symmetric MILPs and MINLPs via a reformulation of the narrowing type [31].

Definition 1 Given a problem $P$, a narrowing $Q$ of $P$ is such that (a) there is a function $\eta: \mathscr{F}(Q) \rightarrow \mathscr{F}(P)$ for which $\eta(\mathscr{G}(Q)) \subseteq \mathscr{G}(P)$, and (b) $Q$ is infeasible only if $P$ is.

The proposed narrowing rests on adjoining some static symmetry breaking inequalities (SSBIs) [38] to the original formulation, i.e. inequalities that are designed to cut some of the symmetric solutions while keeping at least one optimal one. The reformulated problem is then solved by standard software packages such as CPLEX [22] (for MILPs) and COUENNE [4] (for MINLPs, replaced sometimes by BARON [47] on COUENNE's failures). In the same spirit as [31], our reformulation is completely automatic, in the sense that given the original problem we automatically compute the formulation group as well as the narrowing.

With respect to the existing literature about symmetry in mathematical programming, the main contribution of this paper is that of being able to deal with symmetric MINLPs and NLPs, and not just MILPs and Semidefinite Programs (SDPs) as was previously the case $[34,23,19,43,52]$. Moreover, whereas many existing works assume that the formulation group is known in advance, we propose a method for computing the formulation group of a MINLP automatically. The SSBIs we employ for constructing narrowing reformulations hold for every possible group and are well-behaved numerically. We provide computational validation of our ideas by (a) supplying formulation group tables for most of the instances in the MIPLib3 [6], MIPLib2003 [39], GlobalLib [10] and MINLPLib [11] (which also contains MacMINLP [26]); (b) evaluating BB performance on the symmetric instances in the aforementioned libraries, with and without SSBIs.

The rest of this paper is organized as follows. In Sect. 3 we perform a literature review concerning the use of group theoretical methods in mathematical programming. We define several groups linked to a mathematical program in Sect. 4. In Sect. 5 we introduce expression trees and DAGs for representing mathematical functions. We explain in Sect. 6 how to compute a formulation group automatically. Sect. 7 introduces several types of SSBIs and 
some ways to combine them. Computational results validating the proposed approach are given in Sect. 8: these include formulation group tables (Sect. 8.3) as well as results tables (Sect. 8.4).

\section{Notation}

Most of the groups considered in this paper act on vectors in $\mathbb{R}^{n}$ by permuting the components. Permutations act on sets of vectors by acting on each vector in the set. We denote the identity permutation by $e$. We employ standard group nomenclature: $S_{n}, C_{n}$ are the symmetric and cyclic groups of order $n$, and $D_{2 n}$ is the dihedral group of order $n$ (i.e. the group of rotations and reflections of a regular $n$-polygon in the plane). If $G$ is a subgroup of $H$, we write $G \leq H$. If $G, H$ are groups, then the cartesian (set) product $G \times H$ can be endowed with a group structure by defining $(\pi, \sigma)\left(\pi^{\prime}, \sigma^{\prime}\right)=\left(\pi \pi^{\prime}, \sigma \sigma^{\prime}\right)$ for all $(\pi, \sigma),\left(\pi^{\prime}, \sigma^{\prime}\right) \in G \times H$. Two groups $G, H$ are isomorphic (denoted $G \cong H$ ) if there is a group homomorphism $\phi: G \rightarrow H$ (i.e. $\phi$ is such that $\phi\left(\pi \pi^{\prime}\right)=\phi(\pi) \phi\left(\pi^{\prime}\right)$ for all $\pi, \pi^{\prime} \in G$ ) which is both injective and surjective.

For a group $G \leq S_{n}$ and a set $X$ of row vectors, $X G=\{x g \mid x \in X \wedge g \in G\}$; if $Y$ is a set of column vectors, $G Y=\{g y \mid y \in Y \wedge g \in G\}$. If $X=\{x\}$, we denote $X G$ by $x G$ (and similarly $G Y$ by $G y$ if $Y=\{y\}$ ). We say that $G$ fixes $X$ setwise if $X G=X$, and pointwise if $\forall x \in X x g=x$ (similarly for $Y$ - if not otherwise specified, we shall refer to setwise rather than pointwise fixing). We refer to $x G$ as the orbit of $x$ in $G$ (similarly for $G y$ ). In computational group theory literature the notation $\operatorname{orb}(x, G)$ is sometimes employed instead of the more algebraic $x G$. The (setwise) stabilizer $\operatorname{stab}(X, G)$ of a set $X$ with respect to a group $G$ is the largest subgroup $H$ of $G$ that fixes $X$ (i.e. such that $X H=X$ ). For any permutation $\pi \in S_{n}$, let $\Gamma(\pi)$ be the set of its disjoint cycles, so that

$$
\pi=\prod_{\tau \in \Gamma(\pi)} \tau
$$

For a group $G$ and $\pi \in G$ let $\langle\pi\rangle$ be the smallest subgroup of $G$ containing $\pi$, and for a subset $S \subseteq G$ let $\langle S\rangle$ be the smallest subgroup of $G$ containing all elements of $S$ (we also use the terminology subgroup generated by $\pi$ and, respectively, $S$ ), in which case $S$ is a set of generators of $\langle S\rangle$. For any $\pi \in S_{n}$, let $o(\pi)=|\langle\pi\rangle|$ denote the order of $\pi$. If $\pi$ is expressed as a product of disjoint cycles, $o(\pi)$ turns out to be the least common multiple of all the cycle lengths.

Given $B \subseteq\{1, \ldots, n\}, \operatorname{Sym}(B)$ is the symmetric group of all the permutations of elements in $B$. A permutation $\pi \in S_{n}$ is limited to $B$ if it fixes every element outside $B ; \pi$ acts on $B \subseteq\{1, \ldots, n\}$ as a permutation $\rho \in \operatorname{Sym}(B)$ if

$$
\prod_{\tau \in \Gamma(\pi) \cap \operatorname{Sym}(B)} \tau=\rho,
$$

in which case we denote $\rho$ by $\pi[B]$, and call $\rho$ the restriction of $\pi$ to $B$. Because disjoint cycles commute, it follows from the definition that for all $k \in \mathbb{N}, \pi^{k}[B]=(\pi[B])^{k}$. A group $G$ of permutations of $S_{n}$ with generators $\left\{g_{1}, \ldots, g_{s}\right\}$ acts on $B \subseteq\{1, \ldots, n\}$ as $H$ if $\left\langle g_{i}[B]\right| i \leq$ $s\rangle=H$; in this case we denote $H$ by $G[B]$. If $B$ is an orbit of the natural action of $G$ on the integers, then it is easy to show that $G[B]$ is a transitive constituent of $G$, defined [21] as the set of restrictions to $B$ of the elements of $G$ whenever $B$ is an orbit. In general, though, $G[B]$ may not even need to be a subgroup of $G$ : take $G=\langle(1,2)(3,4),(1,3),(4,2)\rangle$ and $B=\{1,2\}$, then $G[B]=\langle(1,2)\rangle \not \leq G$. Let $B, D \subseteq\{1, \ldots, n\}$ with $B \cap D=\emptyset$; if $\pi \in S_{n}$ fixes both $B, D$ setwise, it is easy to show that $\pi[B \cup D]=\pi[B] \pi[D]$. 


\section{Literature review}

We provide here an essential review of group-based methods in mathematical programming, with the notable exceptions of SDP-related results [52] and Constraint Programming (CP) [13] because mostly out of scope - see [38] for more information. Notwithstanding, a technique for automatic symmetry detection in $\mathrm{CP}$ bearing some similarity to the one proposed here can be found in [45]. The existing work may be classified in three broad categories: (a) the abelian group approach proposed by Gomory to write integer feasibility conditions for Integer Linear Programs (ILPs), not reviewed here because out of scope (see [30] for details); (b) symmetry-breaking techniques for specific problems, whose symmetry group can be computed in advance; (c) general-purpose symmetry group computations and symmetrybreaking techniques to be used in BB-type solution algorithms.

Category (b) is possibly the richest in terms of number of published papers. Many types of combinatorial problems exhibit a certain amount of symmetry. Symmetries are usually broken by means of specific branching techniques (e.g. [36]), appropriate global cuts (e.g. [50]) or special formulations $[25,8]$ based on the problem structure. The main limitation of the methods in this category is that they are difficult to generalize and/or to be made automatic.

Category (c) contains three main research streams. The first was established by Margot in the early 2000s [34,35], and is applicable to problems in general form (1) where $x^{L}=$ $0, x^{U}=1$, i.e. Binary Linear Programs (BLPs). Margot [34,38] defines the relaxation group $G^{\mathrm{LP}}(P)$ of a BLP $P$ as:

$$
G^{\mathrm{LP}}(P)=\left\{\pi \in S_{n} \mid c \pi=c \wedge \exists \sigma \in S_{n}(\sigma b=b \wedge \sigma A \pi=A)\right\},
$$

or, in other words, all relabellings of problem variables for which the objective function and constraints are the same. The relaxation group (2) is used to derive effective BB pruning strategies by means of isomorphism pruning and isomorphism cuts local to some selected BB tree nodes (Margot extended his work to general integer variables in [37]). Further results along the same lines, where branching on symmetric nodes at the same level is carried out implicitly (orbital branching), can be obtained for covering and packing problems [43, 44]: if $O$ is an orbit of a certain subgroup of the relaxation group, at each BB node the disjunction $\left(\bigvee_{i \in O} x_{i}=1\right) \vee \sum_{i \in O} x_{i}=0$ induces a feasible division of the search space; orbital branching restricts this disjunction to $x_{h}=1 \vee \sum_{i \in O} x_{i}$ where $h$ is an arbitrary index in $O$. A method for finding the MILP relaxation group (2), based on solving an auxiliary MILP encoding the conditions $\sigma A \pi=A, c \pi=c$ and $\sigma b=b$ in the constraints, was proposed in [29].

The second stream was established by Kaibel et al. in 2007 [23] (also see [16]). Symmetries in the column space (i.e. permutations of decision variables) of binary ILPs having 0-1 constraint matrices are shown to affect the quality of the linear programming bound. Limited only to permutations in cyclic and symmetric group, complete descriptions of orbitopes are provided by means of linear inequalities. Let $x^{\prime}$ be a point in $\{0,1\}^{n}$ (the solution space), with $n=p q$, so that we can arrange the components of $x^{\prime}$ in a matrix $C$. Given a group $G$ and $\pi \in G$, for all 0-1 $p \times q$ matrices $C$ let $\pi C$ be the matrix obtained by permuting the columns of $C$ according to $\pi$. Let $G C$ be the orbit of $C$ under the action of all $\pi \in G, \overline{G C}$ be the lexicographically maximal matrix in $G C$ (ordering matrices by rows first) and $\mathscr{M}_{p q}^{\max }(G)$ be the set of all $\overline{G C}$. Then the full orbitope associated with $G$ is $\operatorname{conv}\left(\mathscr{M}_{p q}^{\max }(G)\right)$. An automatic symmetry detection method for certain orbitopal symmetries that works in linear time is described in [5]. Inspired by the work on orbitopes, E. Friedman recently proposed a similar 
but extended approach [19] leading to the application of fundamental domains (see [38] for a definition of this well-known concept) to symmetry reduction: given a feasible polytope $X \subseteq[0,1]^{n}$ with integral extreme points and a group $G$ acting as an affine transformation on $X$ (i.e. for all $\pi \in G$ there is a matrix $A \in G L(n)$ and an $n$-vector $d$ such that $\pi x=A x+d$ for all $x \in X$ ), a fundamental domain is a subset $F \subset X$ such that $G F=X$.

\section{Groups of a mathematical program}

Given a MINLP $P$ as in (1), the solution group $G^{*}(P)$ of $P$ is defined as $\operatorname{stab}\left(\mathscr{G}(P), S_{n}\right)$, i.e. the group of all permutations of variable indices mapping global optima into global optima. When $P$ is a MILP, $G^{*}(P)$ contains as a subgroup the symmetry group of $P$, defined for MILPs in [38] as the group of permutations mapping feasible solutions into feasible solutions with the same objective function value. Solution groups are hard to compute for a general MINLP (1) because presumably explicit knowledge of $\mathscr{G}(P)$ is needed a priori. We consider the group $\bar{G}_{P}$ that "fixes the formulation" of $P$ :

$$
\begin{aligned}
\bar{G}_{P}= & \left\{\pi \in S_{n} \mid Z \pi=Z \wedge \forall x \in \operatorname{dom}(f) f(x \pi)=f(x) \wedge\right. \\
& \left.\exists \sigma \in S_{m}(\sigma b=b \wedge \forall x \in \operatorname{dom}(g) \sigma g(x \pi)=g(x))\right\} .
\end{aligned}
$$

It is easy to show that $\bar{G}_{P} \leq G^{*}(P)$ : let $\pi \in \bar{G}_{P}$ and $x^{*} \in \mathscr{G}(P) ; x^{*} \pi \in \mathscr{F}(P)$ because $Z \pi=Z$, $g\left(x^{*} \pi\right)=\sigma^{-1} g\left(x^{*}\right)$ and $\sigma^{-1} b=b$; and it has the same function value because $f\left(x^{*} \pi\right)=$ $f\left(x^{*}\right)$ by definition. Thus $\mathscr{G}(P) \pi=\mathscr{G}(P)$ and $\pi \in G^{*}(P)$.

The two most problematic conditions that need testing to ascertain whether a given permutation $\pi$ is in $\bar{G}_{P}$ are:

$$
\begin{aligned}
\forall x \in \operatorname{dom}(f) \quad f(x \pi) & =f(x) \\
\exists \sigma \in S_{m} \forall x \in \operatorname{dom}(g) \quad \sigma g(x \pi) & =g(x) .
\end{aligned}
$$

Since NONLINEAR EQUATIONS (determining if a set of general nonlinear equations has a solution) is an undecidable problem in general [55], such tests are algorithmically infeasible. We therefore assume that for functions $f_{1}, f_{2}: \mathbb{R}^{n} \rightarrow \mathbb{R}$ we have an oracle equal $\left(f_{1}, f_{2}\right)$ that, if it returns true, then $\operatorname{dom}\left(f_{1}\right)=\operatorname{dom}\left(f_{2}\right)$ and $\forall x \in \operatorname{dom}\left(f_{1}\right)\left(f_{1}(x)=f_{2}(x)\right)$, in which case we write $f_{1} \equiv f_{2}$. We remark that we do not ask that the equal oracle returning false should imply $f_{1} \neq f_{2}$ : although this will make equality a stricter notion than it need be (so some pairs of equal functions will not belong to the $\equiv$ relation), it will allow us to implement the oracle efficiently by means of expression trees (see Sect. 5). We can now define the formulation group of a MINLP $P$ as follows:

$$
G_{P}=\left\{\pi \in S_{n} \mid Z \pi=Z \wedge f(x \pi) \equiv f(x) \wedge \exists \sigma \in S_{m}(\sigma g(x \pi) \equiv g(x) \wedge \sigma b=b)\right\} .
$$

The structure of $G_{P}$ depends on the oracle used to implement equality testing. With respect to a trivial oracle always returning false, for example, $G_{P}$ would always only consist of the identity. Because for any function $h, h(x \pi) \equiv h(x)$ implies $h(x \pi)=h(x)$ for all $x \in \operatorname{dom}(h)$, it is clear that $G_{P} \leq \bar{G}_{P}$. Thus, it also follows that $G_{P} \leq G^{*}(P)$.

Although $\bar{G}_{P}$ is defined for any MINLP (1), if $P$ is a BLP, then $\bar{G}_{P}$ is the same group as $G^{\mathrm{LP}}(P)$, as the following result shows.

Proposition 2 Given a problem $P$ as in (1) such that $f, g$ are linear forms, $Z=\{1, \ldots, n\}$ and $x^{L}=\mathbf{0}, x^{U}=\mathbf{1}$, we have $\bar{G}_{P}=G^{\mathrm{LP}}(P)$. 
Proof Let $\pi \in G^{\mathrm{LP}}(P)$; then (a) $c \pi=c$ and (b) $\exists \sigma \in S_{m}$ such that $\sigma b=b$ and $\sigma A b=A$. Let $f(x)=c x$ in (1); then $f(x \pi)=c(x \pi)=(c \pi) x$, and by (a) we have $f(x \pi)=c x=f(x)$. Now let $g(x)=A x$ in (1); then $g(x \pi)=A(x \pi)=(A \pi) x$, and by (b) there is $\sigma \in S_{m}$ such that $\sigma b=b$ and $\sigma A \pi=A$. Thus $\sigma g(x \pi)=\sigma((A \pi) x)=(\sigma A \pi) x=A x=g(x)$, and $\pi \in \bar{G}_{P}$. The implication $\bar{G}_{P} \leq G^{\mathrm{LP}}(P)$ follows directly from the definition (3) if $P$ is a BLP.

\section{A function equality test oracle}

Any mathematical expression consisting of a finite sequence of operator symbols, variable symbols and numerical constants can be represented by an $n$-ary expression tree $[15,14$, $3]$. We consider a finite set $\mathscr{O}$ of operators ordered according to a given order (for example, lexicographically according to their English names); we remark that operators can be unary (such as logarithm, exponential, sine, cosine, etc.), binary (such as fraction, difference, power) or $k$-ary (such as sum and product) for some positive integer $k$. The usual operator precedences, modified by parentheses, apply. Given a function $h(x)$, its expression tree is a directed tree $h=\left(V_{h}, A_{h}\right)$ where $V_{h}$ is partitioned in leaf nodes (labelled with variable symbols from $x_{1}, \ldots, x_{n}$ and numerical constants) and non-leaf nodes (labelled with operator symbols from $\mathscr{O})$, and an $\operatorname{arc}(u, v)$ is in $A_{h}$ if $v$ is an $\operatorname{argument}$ of the operator node $u$. The tree $h$ is constructed recursively as follows: the root of $h$ is the lexicographically smallest operator $\otimes$ of lowest precedence in $h(x)$. Let $h_{1}(x), \ldots, h_{K}(x)$ be the arguments of $\otimes$. Since each $h_{k}(x)$ is a mathematical expression, by induction it is represented by a tree $h_{k}=\left(V_{h_{k}}, A_{h_{k}}\right)$. The vertex set $V_{h}$ is then defined as $\{\otimes\} \cup \bigcup_{k<K} V_{h_{k}}$ and $A_{h}$ as $\bigcup_{k<K}\left(\left(\otimes, h_{k}\right) \cup A_{k}\right)$. In general, expressions need not have unique trees. However, the number of trees corresponding to a given function can be decreased by defining a set of simplification rules ([28], p. 246-247) and an arbitrary argument ordering for each operator (e.g. constants first, then variables in lexicographic ordering, then other operators in the ordered set $\mathscr{O}$ [27]). If $f: \mathbb{R}^{n} \rightarrow \mathbb{R}$, given a vector of values $x^{\prime} \in \mathbb{R}^{n}$, the value $f\left(x^{\prime}\right)$ can be obtained algorithmically by a simple recursive procedure eval $\left(f, x^{\prime}\right)$ on the expression tree $f$ ([28], p. 243), which returns the symbol $\mathrm{NaN}$ (Not a Number) whenever $f\left(x^{\prime}\right)$ is undefined. The equal oracle for two expression trees $f, g$ is defined in Algorithm 1 .

Using Algorithm 1, it is easy to show that if equal $\left(f_{1}, f_{2}\right)=$ true, then $f_{1}(x)=f_{2}(x)$ for all $x$ in the domains of $f_{1}, f_{2}$, whereas the converse is not true (e.g. $\sin (x)=\cos (x+\pi / 2)$ for all $x \in \mathbb{R}$ but the trees corresponding to $\sin (x)$ and $\cos (x+\pi / 2)$ are different). As remarked in Sect. 4 , if equal $\left(f_{1}, f_{2}\right)=$ true we write $f_{1}(x) \equiv f_{2}(x)$ (in this expression there is no need to quantify over $x$, as $\equiv$ is an equality relation between the two trees representing $\left.f_{1}(x), f_{2}(x)\right)$.

Restricted to linear forms, the relation $\equiv$ is the same as equality.

Lemma 3 If $f_{1}, f_{2}$ are linear forms, then $\forall x \in \operatorname{dom}\left(f_{1}\right) f_{1}(x)=f_{2}(x)$ (written $f_{1}=f_{2}$ ) implies $f_{1} \equiv f_{2}$.

Proof Assume $f_{1}=f_{2}$; let $f_{1}(x)=c x$ and $f_{2}(x)=d x$, where $c=\left(c_{1}, \ldots, c_{n}\right), d=\left(d_{1} \ldots, d_{n}\right)$, $x=\left(x_{1}, \ldots, x_{n}\right) \in \mathbb{R}^{n}$. We define the canonical expression tree for the linear form $c x$ by:

$$
\begin{aligned}
V_{c} & =\left\{+, \times_{1}, \ldots, \times_{n}, c_{1}, \ldots, c_{n}, x_{1}, \ldots, x_{n}\right\} \\
A_{c} & =\left\{\left(+, \times_{i}\right),\left(\times_{i}, c_{i}\right),\left(\times_{i}, x_{i}\right) \mid i \leq n\right\}
\end{aligned}
$$

it is easily shown that the canonical tree is unique and that there are finite deterministic algorithms reducing any other expression tree representing $c x$ to the canonical tree (similarly 


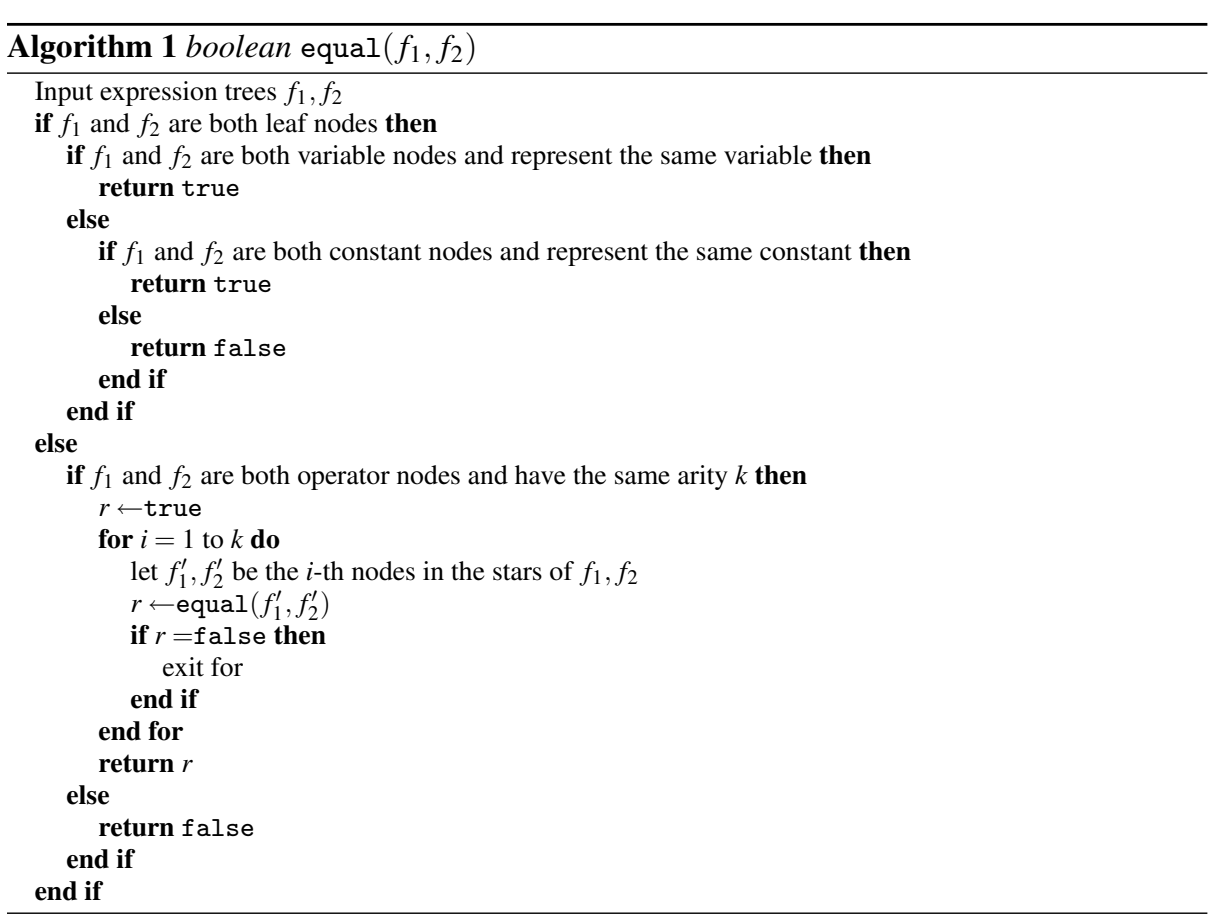

for $d x$ ). Now let $f_{1}(x)=f_{2}(x)$ for all $x \in \mathbb{R}^{n}$; then $\forall x(c x=d x)$, which implies $c=d$, and thus the canonical expression tree for $c$ is identical to the canonical expression tree for $d$. This shows that $f_{1} \equiv f_{2}$.

By Lemma 3 and Prop. 2, given a problem $P$ as in (1) such that $f, g$ are linear forms, $Z=$ $\{1, \ldots, n\}, x^{L}=\mathbf{0}$ and $x^{U}=\mathbf{1}$, we have $G_{P}=G^{\mathrm{LP}}(P)$.

The functions $f, g$ appearing in (1) have the property that their argument list $x$ is the same, so the trees for $f, g_{1}, \ldots, g_{m}$ can share the same variable leaf nodes. This yields a Directed Acyclic Graph (DAG) $D_{P}=\left(V_{P}, A_{P}\right)$ where $V_{P}=V_{f} \cup \bigcup_{i \leq m} V_{g_{i}}$ and $A_{P}=A_{f} \cup$ $\bigcup_{i<m} A_{g_{i}} . D_{P}$ is a DAG representing the mathematical structure of the functions of $P$. It is rooted at the smallest operators of lowest precedence in $f, g_{1}, \ldots, g_{m}$; its leaf nodes are the problem variables and all the problem constants. More comprehensive discussions about expression DAGs and their uses in optimization can be found in $[4,42,48]$.

\section{Automatic computation of the formulation group}

\subsection{Fixed subsets of DAG nodes}

We emphasize the following subsets of $V_{P}$ : the set $\mathscr{S}_{F}$ of all root nodes corresponding to objective functions (in this section we generalize to multi-objective problems although in practice we only consider single objective problems), the set $\mathscr{S}_{C}$ of all root nodes corresponding to constraints, the set $\mathscr{S}_{O}$ of all operator nodes, the set $\mathscr{S}_{K}$ of all constant nodes and the set $\mathscr{S}_{V}$ of all variable nodes. We remark that $\mathscr{S}_{F} \cup \mathscr{S}_{C} \cup \mathscr{S}_{O} \cup \mathscr{S}_{K} \cup \mathscr{S}_{V}=V_{P}$ but the union is not disjoint as $\mathscr{S}_{F} \cup \mathscr{S}_{C} \subseteq \mathscr{S}_{O}$. For a node $v \in \mathscr{S}_{F}$, we denote the optimization 
direction by $d(v)$; for a node $v \in \mathscr{S}_{C}$, we denote the constraint sense by $s(v)$ and the corresponding constraint RHS constant by $b(v)$. For a node $v \in \mathscr{S}_{O}$, we let $\ell(v)$ be the level of $v$ in $D_{P}, \lambda(v)$ be its operator label (operator name) and o $(v)$ be the rank of $v$ in the argument list of its parent node if the latter represents a noncommutative operator, or 1 otherwise. We remark that for nodes in $\mathscr{S}_{O}$ the level in $D_{P}$ is well-defined, as the only nodes in $D_{P}$ with more than one incoming arc are the leaf nodes, and no operator node can be a leaf. For $v \in \mathscr{S}_{K}$, we let $\mu(v)$ be the value of $v$. For $v \in \mathscr{S}_{V}$ we let $r(v)$ be the 2-vector of lower and upper variable bounds for $v$ and $\zeta(v)$ be 1 if $v$ represents an integral variable or 0 otherwise.

We define the relation $\sim$ on $V_{P}$ as follows.

$$
\begin{aligned}
\forall u, v \in V_{P} \quad u \sim v & \Leftrightarrow\left(u, v \in \mathscr{S}_{F} \wedge d(u)=d(v)\right) \\
& \vee\left(u, v \in \mathscr{S}_{C} \wedge s(u)=s(v) \wedge b(u)=b(v)\right) \\
& \vee\left(u, v \in \mathscr{S}_{O} \wedge \ell(u)=\ell(v) \wedge \lambda(u)=\lambda(v) \wedge \mathrm{o}(u)=\mathrm{o}(v)\right) \\
& \vee\left(u, v \in \mathscr{S}_{K} \wedge \mu(u)=\mu(v)\right) \\
& \vee\left(u, v \in \mathscr{S}_{V} \wedge r(u)=r(v) \wedge \zeta(u)=\zeta(v)\right) .
\end{aligned}
$$

It is easy to show that $\sim$ is an equivalence relation on $V_{P}$, and therefore partitions $V_{P}$ into $K$ disjoint subsets $V_{1}, \ldots, V_{K}$.

\subsection{The projection homomorphism}

Let $G \leq S_{n}$ and $\omega$ be a subset of $\{1, \ldots, n\}$. Let $H=\operatorname{Sym}(\omega)$ and define the mapping $\varphi: G \rightarrow H$ by $\varphi(\pi)=\pi[\omega]$ for all $\pi \in G$.

Theorem $4 \varphi$ is a group homomorphism if and only if $G$ stabilizes $\omega$ setwise.

Proof $(\Rightarrow)$ Assume $\varphi$ is a group homomorphism and suppose there is $\sigma \in G$ and $i \in \omega$ such that $\sigma(i)=j \notin \omega$. Take any permutation $\pi \in H$ such that $\pi(i)=k \in \omega, k \neq i$. Then the action of $\pi \sigma$ is to move $i$ to $j$ first (because of $\sigma$ ), and then fix it to $j$ (because of $\pi$ ), which means that $(\pi \sigma)[\omega]$ simply fixes $i$; on the other hand, the action of $\pi[\omega] \sigma[\omega]$ on $i$ is to fix it first (because of $\sigma[\omega]$ ) and then move it to $k$ (because of $\pi[\omega]$ ), hence $\varphi(\pi \sigma) \neq \varphi(\pi) \varphi(\sigma)$, against the assumption. Thus $\sigma(i) \in \omega$ for all $i \in \omega$ and $\sigma \in G$, which implies $G \omega=\omega$.

$(\Leftarrow)$ Assume $G \omega=\omega$ and let $\pi, \sigma \in G$. First, for a single cycle $\gamma$ fixing $\omega$ pointwise, we obviously have $\gamma[\omega]=e$. Now consider two single cycles $\beta, \gamma$ appearing in the disjoint cycle product representation of some permutations of $G$. Since $G$ fixes $\omega$ setwise, either: (1) both $\beta, \gamma \in H$, or (2) one is in $H$ and the other is in $S_{n} \backslash H$, or (3) both are in $S_{n} \backslash H$. For case (1), $\beta, \gamma \in H$ implies $\beta[\omega]=\beta$ and $\gamma[\omega]=\gamma$, which yields $(\beta \gamma)[\omega]=\beta \gamma=\beta[\omega] \gamma[\omega]$. For (2), assuming without loss of generality $\beta \in H$ and $\gamma \notin H$, then $(\beta \gamma)[\omega]=\beta[\omega]=\beta[\omega] e=$ $\beta[\omega] \gamma[\omega]$. For $(3),(\beta \gamma)[\omega]=e=e e=\beta[\omega] \gamma[\omega]$. Thus $\varphi(\beta \gamma)=\varphi(\beta) \varphi(\gamma)$. Next, notice that:

$$
\pi \sigma=\left(\prod_{\tau \in \Gamma(\pi)} \tau\right)\left(\prod_{\tau \in \Gamma(\sigma)} \tau\right)=\prod_{\tau \in \Gamma(\pi) \cup \Gamma(\sigma)} \tau .
$$

Hence,

$$
\begin{aligned}
\varphi(\pi \sigma) & =\varphi\left(\prod_{\tau \in \Gamma(\pi) \cup \Gamma(\sigma)} \tau\right)=\prod_{\tau \in \Gamma(\pi) \cup \Gamma(\sigma)} \varphi(\tau)=\prod_{\tau \in(\Gamma(\pi) \cup \Gamma(\sigma)) \cap H} \tau \\
& =\left(\prod_{\tau \in \Gamma(\pi) \cap H} \tau\right)\left(\prod_{\tau \in \Gamma(\sigma) \cap H} \tau\right)=\varphi(\pi) \varphi(\sigma),
\end{aligned}
$$


which completes the proof.

6.3 Mapping graph automorphisms onto the formulation group

For a digraph $D=(V, A)$, its automorphism group $\operatorname{Aut}(D)$ is the group of vertex permutations $\gamma$ such that $(\gamma(u), \gamma(v)) \in A$ for all $(u, v) \in A$ [46]. Let $G^{\mathrm{DAG}}(P)$ be the largest subgroup of $\operatorname{Aut}\left(D_{P}\right)$ fixing $V_{k}$ for all $k \leq K$ (i.e. containing only vertex permutations $\gamma$ such that $\gamma V_{k}=V_{k}$ for all $\left.i \leq K\right)$. For ease of notation, assume without loss of generality that the vertices of $D_{P}$ are ordered so that for all $j \leq n$, the $j$-th vertex corresponds to the leaf node for variable $x_{j}$ (the rest of the order is not important), i.e. $\mathscr{S}_{V}=\{1, \ldots, n\}$.

Lemma $5 G^{\mathrm{DAG}}(P)$ fixes $\mathscr{S}_{V}$ setwise.

Proof By definition, all permutations of $G^{\mathrm{DAG}}(P)$ fix all $V_{k}$ 's (setwise). In particular, since $\left(u, v \in \mathscr{S}_{V} \wedge r(u)=r(v) \wedge \zeta(u)=\zeta(v)\right)$ implies $u \sim v$, there will be a subset $\mathscr{K}$ of $\{1, \ldots, K\}$ such that $\mathscr{S}_{V}=\bigcup_{k \in \mathscr{K}} V_{k}$. Hence $G^{\mathrm{DAG}}(P) \mathscr{S}_{V}=\mathscr{S}_{V}$ as claimed.

Corollary 6 The map $\varphi: G^{\mathrm{DAG}}(P) \rightarrow \operatorname{Sym}\left(\mathscr{S}_{V}\right)$ given by $\varphi(\gamma)=\gamma\left[\mathscr{S}_{V}\right]$ is a group homomorphism.

Proof By Lemma 5 and Thm. 4.

Theorem $7 \operatorname{Im} \varphi=G_{P}$ groupwise.

Proof We first remark that by Cor. $6, \operatorname{Im} \varphi$ is endowed with a group structure, because $G^{\mathrm{DAG}}(P) / \operatorname{Ker} \varphi \cong \operatorname{Im} \varphi$. In particular, $\operatorname{Im} \varphi$ is a subgroup of $S_{n}$. Now let $\psi: G^{\mathrm{DAG}}(P) \rightarrow$ $\operatorname{Sym}\left(\mathscr{S}_{C}\right)$ be given by $\psi(\gamma)=\gamma\left[\mathscr{S}_{C}\right]$. By an argument similar to that of Lemma $5, G^{\mathrm{DAG}}(P)$ fixes $\mathscr{S}_{C}$ setwise, which implies that $\psi$ is a group homomorphism by Thm. 4. Let $\sigma=\psi(\gamma)$ and $\pi=\varphi(\gamma)$. Because $\gamma$ fixes each equivalence class $V_{k}$, we have $Z \pi=Z, f(x \pi) \equiv f(x)$, $\sigma b=b$ and $\sigma g(x \pi) \equiv g(x)$. Conversely, suppose $\pi \in G_{P}$ and there is no automorphism $\gamma$ of $D_{P}$ fixing all $V_{k}$ 's and such that $\varphi(\gamma)=\pi$. Then either $f(x \pi) \not \equiv f(x)$, or there is no $\sigma \in S_{m}$ such that $\sigma g(x \pi) \equiv g(x)$, or $\psi(\gamma) b \neq b$, contradicting the hypothesis. Thus, $\operatorname{Im} \varphi=G_{P}$ setwise. Since both are subgroups of $S_{n}$, the identity isomorphism shows that $\operatorname{Im} \varphi=G_{P}$ groupwise too.

By Thm. 7, we can automatically generate $G_{P}$ by looking for the largest subgroup of $\operatorname{Aut}\left(D_{P}\right)$ fixing all $V_{k}$ 's. Thus, the problem of computing $G_{P}$ has been reduced to computing the (generators of the) automorphism group of a certain vertex-coloured DAG. This is in turn equivalent to the GRAPH ISOMORPHISM (GI) problem [2]. GI is in NP, but it is not known whether it is in $\mathbf{P}$ or NP-complete. A notion of GI-completeness has therefore been introduced for those graph classes for which solving the GI problem is as hard as solving it on general graphs [51]. Rooted DAGs are GI-complete [7] but there is an $O(N)$ algorithm for solving the GI problem on trees ([46], Ch. 8.5.2).

Corollary 8 If $T^{\prime}$ is a set of group generators of $G^{D A G}(P)$, then $T=\left\{\pi\left[\mathscr{S}_{V}\right] \mid \pi \in T^{\prime}\right\}$ is a set of generators for $G_{P}$. 


\section{Symmetry Breaking Constraints}

In this section we shall discuss the automatic generation of two types of SSBIs, one of which is valid for symmetries in any group $G_{P}$, and the other only holds for the full symmetric group $S_{n}$. Because of their generality and of the usual trade-off between generality and efficacy, the general-purpose SSBIs we propose are not the tightest possible; however, it is their generality that makes their automatic generation feasible (and easy) for all MINLPs. We also propose tighter SSBIs that only hold for $S_{n}$, so that we can only generate them automatically for those instances displaying at least one orbit whose stabilizer is the symmetric group. Some works in the literature [50] suggest using very tight and rather general-purpose SSBIs based on interpreting a 0-1 vector as a base- $k$ expansion of an integer number, with the constraints acting on the latter (also see [38], p. 667). Quite apart from the fact that these SSBIs only hold for integer variables with values in $\{0, \ldots, k\}$ (so they would not be applicable to continuous NLPs), it is well known that such SSBIs are badly scaled; so that although the corresponding narrowing is formally well-defined and symmetry-free, it is often much more difficult to solve correctly, in practice, than the original problem. We therefore limit our attention to SBCs that are numerically well behaved.

We first give a formal definition of SSBIs that makes them depend on a group rather than a set of solutions.

Definition 9 Given a permutation $\pi \in S_{n}$ acting on the component indices of the vectors in a given set $X \subseteq \mathbb{R}^{n}$, the constraints $g(x) \leq 0$ (that is, $\left\{g_{1}(x) \leq 0, \ldots, g_{q}(x) \leq 0\right\}$ ) are symmetry breaking constraints (SBCs) with respect to $\pi$ and $X$ if there is $y \in X$ such that $g(y \pi) \leq 0$. Given a group $G, g(x) \leq 0$ are SBCs w.r.t $G$ and $X$ if there is $y \in X G$ such that $g(y) \leq 0$.

If there are no ambiguities as regards $X$, we simply say "SBCs with respect to $\pi$ " (respectively, $G)$. In most cases, $X=\mathscr{G}(P)$. The following facts are easy to prove.

1. For any $\pi \in S_{n}$, if $g(x) \leq 0$ are SBCs with respect to $\pi, X$ then they are also SBCs with respect to $\langle\pi\rangle, X$.

2. For any $H \leq G$, if $g(x) \leq 0$ are SBCs with respect to $H, X$ then they are also SBCs with respect to $G, X$.

3. Let $g(x) \leq 0$ be SBCs with respect to $\pi \in S_{n}, X \subseteq \mathbb{R}^{n}$ and let $B \subseteq\{1, \ldots, n\}$. If $g(x) \equiv$ $g(x[B])$ (i.e., if the constraints $g$ only involve variable indices in $B$ ) then $g(x) \leq 0$ are also SBCs with respect to $\pi[B], X[B]$.

As regards Fact 3, if $g(x) \equiv g(x[B])$ we denote the SBCs $g(x) \leq 0$ by $g[B](x) \leq 0$; if $B$ is the domain of a permutation $\alpha \in \operatorname{Sym}(B)$, we also use the notation $g[\alpha](x) \leq 0$.

Example 10 Let $y=(1,1,-1), X=\{y\}$ and $\pi=(1,2,3)$; then $\left\{x_{1} \leq x_{2}, x_{1} \leq x_{3}\right\}$ are SBCs with respect to $\pi$ and $X$ because $y \pi$ satisfies the constraints. The inequalities $\left\{x_{1} \leq x_{2}, x_{2} \leq\right.$ $\left.x_{3}\right\}$ are SBCs with respect to $S_{3}$ and $X$ because $(-1,1,1)=y(1,2,3) \in X S_{n}$, but not with respect to to $\langle(2,3)\rangle$ and $X$ because $X\langle(2,3)\rangle=\{y, y(2,3)\}=\{(1,1,-1),(1,-1,1)\}$ and neither vector satisfies the constraints.

We use SBCs to yield narrowings of the original problem $P$.

Theorem 11 If $g(x) \leq 0$ are $S B C$ s for any subgroup $G$ of $G_{P}$ and $\mathscr{G}(P)$, then the problem $Q$ obtained by adjoining $g(x) \leq 0$ to the constraints of $P$ is a narrowing of $P$.

Proof By Defn. 1, we must provide a map $\mathscr{G}(Q) \rightarrow \mathscr{G}(P)$ and show that if $P$ is feasible then $Q$ is feasible. Assume $\mathscr{F}(P) \neq \emptyset$; then $\mathscr{G}(P) \neq \emptyset$. By definition of SBCs, there is $y \in \mathscr{G}(P) G$ 
such that $g(y) \leq 0$. Since $G \leq G_{P} \leq G^{*}(P)=\operatorname{stab}\left(\mathscr{G}(P), S_{n}\right)$, it follows that $\mathscr{G}(P) G=\mathscr{G}(P)$, so that $y \in \mathscr{G}(P)$. Thus, $y$ satisfies the constraints of $P$ and also $g(y) \leq 0$, which means that $y \in \mathscr{F}(Q)$, as required. Now, let $\eta$ be the identity map; since $\mathscr{F}(Q) \neq 0$ it follows that $\mathscr{G}(Q)$ contains at least one element $x$. Since $\mathscr{F}(Q) \subseteq \mathscr{F}(P)$ (because $Q$ is as $P$ with additional constraints) and the objective functions of $P, Q$ are equal, $\eta(x)=x \in \mathscr{G}(P)$.

We now describe a way to combine SBCs. Since adjoining more constraints to a formulation results into a smaller feasible region and fewer optima, combined SBCs should yield better narrowings.

Theorem 12 Let $\omega, \theta \subseteq\{1, \ldots, n\}$ be such that $\omega \cap \theta=\emptyset$. Consider $\rho, \sigma \in G_{P}$, and let $g[\omega](x) \leq 0$ be SBCs w.r.t. $\rho, \mathscr{G}(P)$ and $h[\theta](x) \leq 0$ be SBCs w.r.t. $\sigma, \mathscr{G}(P)$. If $\rho[\omega], \sigma[\theta] \in$ $G_{P}[\omega \cup \theta]$ then the system of constraints $c(x) \leq 0$ consisting of $g[\omega](x) \leq 0$ and $h[\theta](x) \leq 0$ is an $S B C$ system for $\rho \sigma$.

Proof Let $y \in \mathscr{G}(P)$. Since $g[\omega](x)$ only depends on variable indices in $\omega, g[\omega](y \rho[\omega]) \leq 0$. Likewise, $h[\theta](y \sigma[\theta]) \leq 0$. The fact that $\rho[\omega], \sigma[\theta] \in G_{P}[\omega \cup \theta]$ implies that $\rho[\omega \cup \theta]=$ $\rho[\omega]$ and $\sigma[\omega \cup \theta]=\sigma[\theta]$, and in turn that $\rho[\theta]=\sigma[\omega]=e$. Since $\sigma$ fixes $\omega$ pointwise, the action of $\rho \sigma$ on $\omega$ reduces to the action of $\rho$ on $\omega$, and similarly for $\rho$ and $\theta$, i.e. $(\rho \sigma)[\omega]=$ $\rho[\omega]$ and $(\rho \sigma)[\theta]=\sigma[\theta]$. Thus, $g[\omega](y \rho \sigma)=g[\omega](y(\rho \sigma)[\omega])=g[\omega](y \rho[\omega]) \leq 0$ and $h[\theta](y \rho \sigma)=h[\theta](y(\rho \sigma)[\theta])=h[\theta](y \sigma[\theta]) \leq 0$, hence $c(y \rho \sigma) \leq 0$ as claimed.

Thm. 12 can easily be extended to sets of subsets of $\{1, \ldots, n\}$ where the required conditions hold pairwise.

\subsection{SBCs from orbits}

Consider the set $\Omega$ of (nontrivial) orbits of the natural action of $G_{P}$ on $\{1, \ldots, n\}$. We pave the way for applying Thm. 12 to adjoin SBCs arising from different orbits. Since $G_{P}$ acts transitively on each orbit $\omega \in \Omega$, for all $i \neq j \in \omega$ there is at least one permutation in $G_{P}$ mapping $i$ to $j$. Let $M^{i j} \subseteq G_{P}$ be the set of all such permutations. Let $\omega, \theta \in \Omega$ be such that:

1. $\forall i \neq j \in \omega \exists \rho \in M^{i j}$ s.t. $\operatorname{gcd}(o(\rho[\omega]), o(\rho[\theta]))=1$; let $\tilde{R}$ be the set of all such $\rho$

2. $\forall i \neq j \in \theta \exists \sigma \in M^{i j}$ s.t. $\operatorname{gcd}(o(\sigma[\omega]), o(\sigma[\theta]))=1$; let $\tilde{S}$ be the set of all such $\sigma$.

Lemma 13 For all $\rho \in \tilde{R}$ and $\sigma \in \tilde{S}, \rho[\omega], \sigma[\theta] \in G_{P}[\omega \cup \theta]$.

Proof Let $r=o(\rho[\theta])$.

$$
\begin{aligned}
\rho^{r}[\omega \cup \theta] & =\rho^{r}[\omega] \rho^{r}[\theta] \text { as } \omega, \theta \text { are (setwise fixed) orbits of } G_{P} \\
& =\rho[\omega]^{r} \rho[\theta]^{r} \text { by definition (Sect. 2) } \\
& =\rho[\omega]^{r} \quad \text { because } r \text { is the order of } \rho[\theta] .
\end{aligned}
$$

Now $\left\langle\rho[\omega]^{r}\right\rangle=\langle\rho[\omega]\rangle$ because $\operatorname{gcd}(o(\rho[\omega]), r)=1$ by definition. Thus there is a positive integer $t$ such that $\rho[\omega]^{r t}=\rho[\omega]$, which means that $\rho[\omega]=\rho[\omega]^{r t}=\rho^{r}[\omega]^{t}=\rho^{r}[\omega \cup \theta]^{t} \in$ $G_{P}[\omega \cup \theta]$. The argument for $\sigma[\theta]$ is similar.

Lemma 13 and Thm. 12 establish the following.

Corollary 14 If $g[\omega](x) \leq 0$ are SBCs w.r.t. some $\rho \in \tilde{R}$ and $h[\theta](x) \leq 0$ are SBCs w.r.t. some $\sigma \in \tilde{S}$, the union of both systems is an $S B C$ system for $\rho \sigma$. 
We now propose general-purpose $\mathrm{SBCs}$, valid for $G_{P}$, which can be derived from any of its orbits.

Proposition 15 Let $\omega \in \Omega$. The constraints

$$
\forall j \in \omega \backslash\{\min \omega\} \quad x_{\min \omega} \leq x_{j} .
$$

are $S B C s$ with respect to $G_{P}$.

Proof Let $y \in \mathscr{G}(P)$. Since all groups act transitively on each orbit, there is $\pi \in G_{P}$ mapping $\min y[\omega]$ to $y_{\min \omega}$. Thus, $y \pi$ satisfies (5).

If there is $\omega \in \Omega$ such that the action of $G_{P}$ on it is the symmetric group on $\omega$, stronger SBCs than (5) hold. Let $\omega^{-}=\omega \backslash\{\max \omega\}$, and for each $j \in \omega^{-}$let $j^{+}=\min \{h \in \omega \mid h>j\}$ be the successor of $j$ in $\omega$.

Proposition 16 Provided $G_{P}[\omega]=\operatorname{Sym}(\omega)$, the following constraints:

$$
\forall j \in \omega^{-} \quad x_{j} \leq x_{j^{+}}
$$

are $S B C s$ with respect to $G_{P}$.

Proof Let $y \in \mathscr{G}(P)$. Since $G_{P}[\omega]=\operatorname{Sym}(\omega)$, there is $\pi \in G_{P}$ such that $(y \pi)[\omega]$ is ordered by $\leq$. Therefore, $y \pi$ is feasible with respect to the constraints $\forall j \in \omega^{-} x_{j} \leq x_{j^{+}}$, which yields the result.

By Cor. 14, any set of SBC systems with respect to transitive constituents of $G_{P}$ whose corresponding orbits verify Conditions 1-2 (top of this subsection) pairwise is a system of SBCs w.r.t. $G_{P}$.

Proposition 17 Let $\omega, \theta \in \Omega$ and assume $G_{P}[\omega \cup \theta]$ contains a subgroup $H \cong C_{|\omega|} \times C_{|\theta|}$ such that $H[\omega] \cong C_{|\omega|}$ and $H[\theta] \cong C_{|\theta|}$. Then $\omega, \theta$ satisfy Conditions 1-2.

Proof Let $\rho \in G_{P}$ such that $\rho[\omega \cup \theta] \in H$ be chosen so that $\langle\rho[\omega]\rangle=H[\omega] \cong C_{|\omega|}$ and $\rho[\theta]=e$. Then for all $i \neq j \in \omega$ there is an integer $k$ such that $\rho^{k}$ maps $i$ to $j$ and fixes $\theta$, and hence $\rho^{k} \in M^{i j} ; \rho[\theta]=e$ ensures $\operatorname{gcd}(o(\rho[\omega]), o(\rho[\theta]))=1$. The argument for $\theta$ is similar.

Proposition 18 Let $\omega, \theta \in \Omega$ and assume that $G_{P}[\omega \cup \theta]$ contains a subgroup $H$ such that $H[\omega] \cong C_{|\omega|}$ and $H[\theta] \cong C_{|\theta|}$. If $\operatorname{gcd}(|\omega|,|\theta|)=1$ then $\omega, \theta$ satisfy Conditions 1-2.

Proof Let $\rho \in G_{P}$ such that $\rho[\omega \cup \theta] \in H$ be chosen so that: (a) $\langle\rho[\omega]\rangle=H[\omega] \cong C_{|\omega|}$; (b) there is a single cycle $\alpha \in H[\theta]$ having length $|\theta|$ and an integer $l$ such that $\rho[\theta]=\alpha^{l}$. Hence $s=o(\rho[\theta])$ divides $|\theta|$. Since $o(\rho[\omega])=|\omega|$ and $\operatorname{gcd}(|\omega|,|\theta|)=1,\left\langle\rho^{s}[\omega]\right\rangle \cong\langle\rho[\omega]\rangle$. Thus, for all $i \neq j \in \omega$ there is an integer $k$ such that $\left(\rho^{s}\right)^{k}=\rho^{s k}$ maps $i$ to $j$, and $\rho^{s k}[\theta]=\rho[\theta]^{s k}=$ $\left(\rho[\theta]^{s}\right)^{k}=e^{k}=e$. Thus $\tau=\rho^{s k}$ is in $M^{i j}$ and $\operatorname{gcd}(o(\tau[\omega]), o(\tau[\theta]))=1$. The argument for $\theta$ is similar.

Since by Sect. 6 we can obtain the set $T$ of generators of $G_{P}$, it is possible to compute the set of orbits $\Omega$ in time $O\left(n|T|+n^{2}\right)$ [12]. There are polynomial-time algorithms for testing group membership and subgroup inclusion [49]; algorithms for dealing with the transitive constituent homomorphism $\varphi$ usually rest on the Schreier-Sims method for computing group generators (of which some implementations as a nearly linear-time Monte Carlo algorithm exist). Thus, deriving SBCs as per Prop. 15 and combining them using Prop. 18 are tasks whose algorithmic implementation is practically feasible. 
7.2 Generating SBCs automatically

We aim to test two different approaches. In the first one, we simply pick the largest orbit, verify it contains a subgroup $C_{|\omega|}$, and adjoin the corresponding SBCs (5) to the original problem. In the second, we attempt to use Prop. 17 and 18 in order to adjoin SBCs (of type (6) if possible) from several orbits. Since (5) only impose a minimum element within a set of values, whereas (6) imposes a whole total order, the latter should yield a tighter narrowing than the former, and we expect a tight narrowing to be easier to solve by BB than a slack one. This is not always true in practice, however, because narrowing constraints may have some adverse effects too, such as making each BB node relaxation longer to solve and affecting the choice of branching variable and/or branching point.

A set $\omega \subseteq\{1, \ldots, n\}$ is a block for $G$ if $\forall g \in G(\omega g=\omega \vee \omega g \cap \omega=\emptyset)$. A group $G$ is primitive if its only blocks are trivial (i.e. $\emptyset$, singletons and $\{1, \ldots, n\}$ ). There are practically fast algorithms for testing groups for primitivity. Let $\omega \in \Omega$ be a nontrivial orbit of $G_{P}$, let $T$ be the set of generators of $G_{P}$ constructed as per Cor. 8, and for any $\omega \subseteq\{1, \ldots, n\}$ let $T[\omega]=\{\pi[\omega] \mid \pi \in T\}$. We first remark that if $T[\omega]$ contains a cycle of length $|\omega|$, then $C_{|\omega|} \leq G_{P}[\omega]$; this provides a practical way of testing the hypotheses of Propositions 17 and 18. The following result can be used for testing the hypothesis of Prop. 16.

Proposition 19 If $G_{P}[\omega]$ is primitive and $T[\omega]$ contains a transposition (i.e. a cycle of length 2$), G_{P}[\omega]=\operatorname{Sym}(\omega)$.

Proof By [53], Thm. 13.3.

Naturally, if $G_{P}[\omega]=\operatorname{Sym}(\omega)$ then $C_{|\omega|} \leq G_{P}[\omega]$, so Prop. 19 can also be used to test the hypotheses of Prop. 17 and 18.

In practice, we form a subset $\Lambda \subseteq \Omega$ of orbits which satisfy the hypotheses of Prop. 18 pairwise. Then, for each orbit $\omega$ in $\Lambda$ we further verify whether $G_{P}[\omega]$ satisfies the hypotheses of Prop. 16 or not. Accordingly, for each orbit in $\Lambda$, we either output SBCs (6) or (5). We attempt to construct $\Lambda$ so that it generates as many added constraints as possible, in the hope of yielding a significantly smaller feasible region. We adopt a greedy approach on the orbit length (Alg. 2).

\section{Computational results}

We report computational results of two kinds. We first attempt to determine a closed form description of $G_{P}$ for all the considered instances (Tables 2-4). Secondly, we compare BB performances on the original and reformulated problems. We remark that our symmetry breaking efforts are limited to the adjoining of static constraints to the formulation (rather than employing dynamic symmetry breaking techniques [38]): with static techniques only, it is not so clear that the proposed approach helps in solving general MILPs, although we have interesting results for some selected instances. The performance on NLPs/MINLPs, on the other hand, is much better. Part of the reason for this is that MILP solvers are technically much more advanced than their NLP/MINLP counterparts — and our MILP solver of choice already contains some symmetry exploitation devices. The good results obtained on MILPs using dynamic symmetry breaking techniques [34,17,44], however, point to the fact that the type of automatic symmetry detection proposed in this paper might be complemented by dynamic symmetry breaking techniques and applied to MILPs quite successfully. This will make the object of further investigations. 


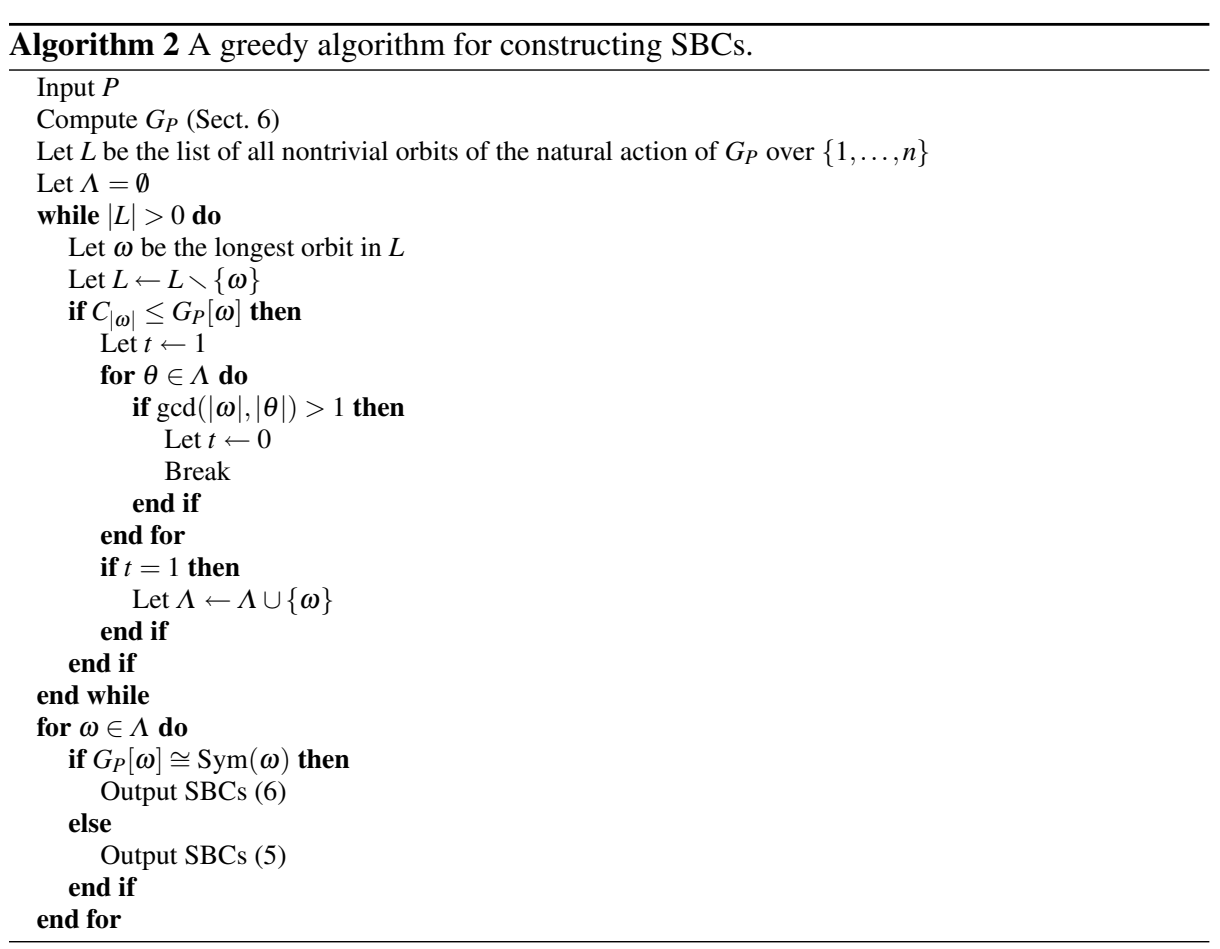

We employ two types of reformulations: Narrowingl is obtained by adjoining (5) for the longest orbit to the original formulation; Narrowing2 adjoins the SBCs returned by Alg. 2. The BB solvers employed are: CPLEX 11.01 [22] for the MILP instances and CouENNE [4] for NLP and MINLP instances; since COUENNE is a relatively young solver, and not yet totally stable, BARON [47] was used whenever COUENNE failed. The solution statistics are:

1. the objective function value of the incumbent

2. the seconds of user CPU time taken (meaningful when below the 7200s limit)

3. the gap still open at termination

4. the number of BB nodes closed and those still on the tree at termination.

A first round of tests compares the statistics after two hours of computation time (per instance). In a second round of tests, we perform the same comparison with different termination criteria on a meaningful subset of instances. All results have been obtained on one 2.4GHz Intel Xeon CPU of a computer with 8 GB RAM (shared by 3 other similar CPUs) running Linux.

\subsection{Implementation}

We implemented two software systems: the first, symmgroup, computes an explicit description of the formulation group structure. The second, reformulate, implements Alg. 2 and produces a reformulated instance ready to be solved. The algorithm that computes the explicit description of a group structure given its generators has exponential worst-case complexity and is in practice quite slow, whereas reformulating entails computing the orbits 
from the generators, computing a group action on an orbit, verifying whether a generator has a certain length, and verifying whether a given group is primitive (all polynomialtime and also practically fast algorithms [49]). Thus, we were not always able to find the group description although we were able to reformulate the original problem to the correct narrowing. The implementation of symmgroup and reformulate is similar up to the stage where the group generators are computed. Both first call AMPL [18] to parse the instance; the ROSE Reformulation/Optimization Software Engine [32] AMPL-hooked solver is then called (with ROSE's Rsymmgroup reformulator) to produce a file representation of the problem expression DAG. This is then fed into nauty's [41,40] dreadnaut shell to efficiently compute the generators of $\operatorname{Aut}\left(D_{P}\right)$ (see Sect. 6). A system of shell scripts and Unix tools parses the nauty output to form a valid GAP [20] input. At this stage, symmgroup uses GAP's StructureDescription command to output the formulation group description, whereas reformulate uses a purpose-built GAP code that simply outputs SBCs (5) relating to the longest orbit (Narrowing1) or implementing Alg. 2 (Narrowing2).

\subsection{Test set}

Our test set consists of almost all the instances in the best known mathematical programming instance libraries: MIPLib3 [6], MIPLib2003 [39] (containing MILPs), GlobalLib [10] (containing NLPs) and MINLPLib [11] (containing MINLPs). We have not tested some of the largest instances (listed in Table 5) because of RAM and CPU time limitations. Our test set consists of a grand total of 669 instances partitioned in the different libraries as given in Table 1 - this table also reports the number of instances whose formulation have nontrivial groups. The instance sizes can be found in the online appendix.

\begin{tabular}{|l|r|rr|}
\hline Library & Instances & Nontrivial $G_{P}$ & \% of library \\
\hline miplib3 & 62 & 22 & $35.4 \%$ \\
miplib2003 $\backslash$ miplib3 & 20 & 7 & $35.0 \%$ \\
globallib & 390 & 58 & $14.8 \%$ \\
minlplib & 197 & 32 & $16.2 \%$ \\
Total & $\mathbf{6 6 9}$ & $\mathbf{1 1 2}$ & $\mathbf{1 6 . 7} \%$ \\
\hline
\end{tabular}

Table 1 Instance libraries statistics.

\subsection{Group tables}

In Table 2 we report formulation groups for all (MILP) instances of the MIPLib3 and MIPLib2003 libraries. In Table 3 we report formulation groups for all (NLP) instances of the GlobalLib library. In Table 4 we report formulation groups for all (MINLP) instances of the MINLPLib library. We remark that all group tables have been compiled with the AMPL presolver disabled. Since the group depends on the formulation rather than the problem itself, the AMPL presolver has an impact on the group structure. This raises an interesting question for future research: determining the exact reformulation of $P$ yielding the formulation group with tightest associated SBCs (a meaningful simplification might call for the reformulation yielding the largest formulation group). An equally interesting question is that of deciding 
whether a given problem instance has a formulation whose group is equal to the solution group.

Critical failures were due to excessive RAM or CPU usage on the part of nauty. Noncritical failures, due to GAP excessive RAM requirements, imply that an explicit description of the group structure is missing but the group generators are provided (so it is possible to reformulate the problem nonetheless). Computational times are not reported in Tables 2-4 because a large share of the total CPU time taken to compute the group structure was taken by GAP's StructureDescription command. Since this was only necessary to compute the tables, but not to reformulate the instances, CPU times at this stage would not be indicative (the CPU time taken to reformulate the instances is reported in Tables 6-9). Just to give a rough idea, compiling all the tables took 7 days of computation, with a significant fraction of the CPU time being taken by all the arki- instances.

MIPLib3 1/2
\begin{tabular}{|l|l|}
\hline Instance & $G_{P}$ \\
\hline air03 & $\left(C_{2}\right)^{13}$ \\
arki001 & $S_{48}$ \\
blend2 & $S_{9}$ \\
enigma & $C_{2}$ \\
fiber & $C_{2}$ \\
gen & $C_{2}$ \\
mas74 & $\left(C_{2}\right)^{2}$ \\
mas76 & $\left(C_{2}\right)^{2}$ \\
misc03 & $S_{3}$ \\
misc06 & $\left(S_{5}\right)^{3}$ \\
misc07 & $S_{3}$ \\
mitre & $\left(C_{2}\right)^{7}$ \\
mkc ${ }^{a}$ & $\langle 193$ generators $\rangle$ \\
noswot & $C_{2}$ \\
p0201 & $\left(C_{2}\right)^{2}$ \\
p2756 & $\left(C_{2}\right)^{29}$ \\
\hline
\end{tabular}

a GAP RAM failure.

MIPLib3 2/2
\begin{tabular}{|l|l|}
\hline Instance & $G_{P}$ \\
\hline qiu & $C_{2} \times S_{4}$ \\
rgn & $S_{5}$ \\
rout & $S_{5}$ \\
seymour & $\langle 216$ generators $\rangle$ \\
stein $27^{d}$ & $\left(\left(C_{3}\right)^{3} \ltimes P S L(3,3)\right) \ltimes C_{2}$ \\
swath $^{d}$ & $\langle 461$ generators $\rangle$ \\
\hline All other & 1 \\
\hline
\end{tabular}

MIPLib2003 $\backslash$ MIPLib3
\begin{tabular}{|l|l|}
\hline Instance & $G_{P}$ \\
\hline glass4 & $C_{2}$ \\
mzzv11 & $\left(C_{2}\right)^{155}$ \\
mzzv42z & $\left(C_{2}\right)^{110}$ \\
opt1217 & $C_{2}$ \\
protfold & $\left(C_{2}\right)^{2}$ \\
timtab1 & $C_{2}$ \\
timtab2 & $C_{2}$ \\
\hline All other & 1 \\
\hline
\end{tabular}

Table 2 MILP instances and formulation groups. The group labelled $\operatorname{PSL}(3,3)$ is the projective special linear group of order 3 on $F_{3}$.

It is worth mentioning (thanks to one of the referees for pointing this out) that the stein45 instance in MIPLib3 has a trivial symmetry group due to an input error of its contributor J. Gregory, as verified by our code. The real Steiner triple incidence matrix actually has significant symmetry.

\subsection{Results tables}

In this section we present comprehensive results tables. Their purpose is to show that breaking symmetry in general helps, specially on NLP/MINLPs. As explained above, we compare the performance of various BB algorithms solving the original problem and two types of narrowings (Narrowing1, adjoining SBCs (5) for the longest orbit; and Narrowing2, adjoining the SBCs output by Alg. 2).

The kind of pattern we notice from the first round of tests (Tables 6-9 - symmetric instances solved with a $2 \mathrm{~h}$ CPU time limit) is twofold. Firstly, more instances are solved faster 
GlobalLib 1/2

\begin{tabular}{|l|l|}
\hline Instance & $G_{P}$ \\
\hline arki0002 & $\left(S_{6}\right)^{2}$ \\
arki0003 & $C_{2}$ \\
arki0008 & $S_{50}$ \\
arki0009 & $\left(S_{5}\right)^{10} \times S_{9} \times S_{11}$ \\
arki0010 & $\left(S_{5}\right)^{5} \times S_{9} \times S_{11}$ \\
arki0011 & $\left(C_{2}\right)^{15} \times S_{3} \times\left(S_{9}\right)^{3} \times S_{20}$ \\
arki0012 & $\left(C_{2}\right)^{15} \times S_{3} \times\left(S_{9}\right)^{3} \times S_{11}$ \\
arki0013 & $\left(C_{2}\right)^{15} \times S_{3} \times\left(S_{9}\right)^{3} \times S_{20}$ \\
arki0014 & $\left(C_{2}\right)^{15} \times S_{3} \times\left(S_{9}\right)^{3} \times S_{20}$ \\
arki0016 & $S_{5}$ \\
elec100 & $S_{3}$ \\
elec25 & $S_{3}$ \\
elec50 & $S_{3}$ \\
ex14_1_5 & $S_{4}$ \\
ex2_1_3 & $C_{2}$ \\
ex5__5 & $S_{3}$ \\
ex6_1_1 & $C_{2}$ \\
ex6__3 & $C_{2}$ \\
ex6__10 & $C_{2}$ \\
ex6__12 & $C_{2}$ \\
ex6__13 & $C_{2}$ \\
ex6__14 & $C_{2}$ \\
ex6_2_5 & $C_{2}$ \\
ex6_2_7 & $S_{3}$ \\
ex6_2_9 & $C_{2}$ \\
ex8_1_6 & $C_{2}$ \\
ex8_2_1 & $\left(S_{4}\right)^{4} \times S_{8}$ \\
ex8_2_2 & $\langle 465$ generators $\rangle$ \\
ex8_2_4 & $\left(S_{4}\right)^{4} \times S_{8}$ \\
\hline
\end{tabular}

GlobalLib 2/2

\begin{tabular}{|l|l|}
\hline Instance & $G_{P}$ \\
\hline ex8_2_5 & $\langle 602$ generators $\rangle$ \\
ex8_3_10 & $S_{5}$ \\
ex8_3_11 & $S_{5}$ \\
ex8_3_12 & $S_{5}$ \\
ex8_3_13 & $S_{5}$ \\
ex8_3_14 & $S_{5}$ \\
ex8_3_1 & $S_{5}$ \\
ex8_3_2 & $S_{5}$ \\
ex8_3_3 & $S_{5}$ \\
ex8_3_4 & $S_{5}$ \\
ex8_3_5 & $S_{5}$ \\
ex8_3_6 & $S_{5}$ \\
ex8_3_7 & $S_{5}$ \\
ex8_3_8 & $S_{5}$ \\
ex8_3_9 & $S_{5}$ \\
ex8_4_6 & $S_{3}$ \\
ex9_1_10 & $C_{2}$ \\
ex9_1_8 & $C_{2}$ \\
ex9_2_6 & $C_{2} \times D_{8}$ \\
ganges & $\left(C_{2}\right)^{6} \times\left(S_{3}\right)^{2}$ \\
gangesx & $\left(C_{2}\right)^{6} \times\left(S_{3}\right)^{2}$ \\
korcge & $\left(C_{2}\right)^{2}$ \\
maxmin & $C_{2}$ \\
st_e18 & $\left(C_{2}\right)^{2}$ \\
st_e39 & $\left(C_{2}\right)^{2}$ \\
st_fp3 & $\left(C_{2}\right)^{2}$ \\
st_rv9 & $\left(C_{2}\right)^{10}$ \\
torsion50 & $C_{2}$ \\
turkey & $\left(C_{2}\right)^{4}$ \\
\hline All other & 1 \\
\hline &
\end{tabular}

${ }^{a}$ GAP RAM failure.

${ }^{a}$ GAP RAM failure.

Table 3 NLP instances and formulation groups.

MINLPLib $1 / 2$
\begin{tabular}{|l|l|}
\hline Instance & $G_{P}$ \\
\hline cecil_13 & $\left(C_{2}\right)^{30}$ \\
deb7 & $S_{10}$ \\
deb8 & $S_{10}$ \\
deb9 & $S_{10}$ \\
elf & $S_{3}$ \\
gastrans & $\left(C_{2}\right)^{2}$ \\
gear & $D_{8}$ \\
gear2 & $D_{8}$ \\
gear3 & $D_{8}$ \\
gear4 & $D_{8}$ \\
hmittelman & $C_{2}$ \\
lop97ic & $\left(C_{2}\right)^{2}$ \\
lop97icx & $\left(C_{2}\right)^{7} \times S_{762}$ \\
nuclear14 & $S_{6}$ \\
nuclear24 & $S_{6}$ \\
nuclear25 & $S_{5}$ \\
nuclear49 & $S_{7}$ \\
\hline
\end{tabular}

MINLPLib $2 / 2$
\begin{tabular}{|l|l|}
\hline Instance & $G_{P}$ \\
\hline nuclearva & $S_{3}$ \\
nuclearvb & $S_{3}$ \\
nuclearvc & $S_{3}$ \\
nuclearvd & $S_{3}$ \\
nuclearve & $S_{3}$ \\
nuclearvf & $S_{3}$ \\
nvs09 & $S_{10}$ \\
product & $\langle 150$ generators $\rangle$ \\
product2 & $\langle 561$ generators $\rangle$ \\
risk2b & $\left(C_{2}\right)^{5} \times\left(S_{3}\right)^{11} \times S_{5} \times\left(S_{6}\right)^{2} \times\left(S_{13}\right)^{3}$ \\
super1 & $\left(C_{2}\right)^{8} \times\left(S_{3}\right)^{4}$ \\
super2 & $\left(C_{2}\right)^{10} \times\left(S_{3}\right)^{2}$ \\
super3 & $\left(C_{2}\right)^{9} \times\left(S_{3}\right)^{2}$ \\
super3t & $\left(C_{2}\right)^{9} \times\left(S_{3}\right)^{2}$ \\
synheat & $S_{4}$ \\
\hline All other & 1 \\
\hline
\end{tabular}

$a$ GAP RAM failure.

Table 4 MINLP instances and formulation groups. 


\begin{tabular}{|l|l|}
\hline Library & Instances \\
\hline MIPLib3 & - \\
\hline MIPLib2003 & ds, momentum3, msc98-ip, sp97ar, stp3d \\
\hline \multirow{2}{*}{ GlobalLib } & $\begin{array}{l}\text { arki0005, arki0006, arki0007, arki0018, } \\
\text { arki0023, arki0024, elec200, ex8_2_3, } \\
\text { jbearing100, minsurf100, torsion100 }\end{array}$ \\
\hline \multirow{3}{*}{ MINLPLib } & $\begin{array}{l}\text { detf1, dosemin2d, dosemin3d, eg_all_s, } \\
\text { eg_disc_s, eg_disc2_s, eg_int_s, mbtd, } \\
\text { nuclear104, nuclear10b, qap }\end{array}$ \\
\hline
\end{tabular}

Table 5 Excessively large instances (nauty RAM or CPU failures during reformulation).

in the narrowing SBC reformulations than in the original problem. Secondly, whereas those instances that are solved faster without SBCs only scrape off a minor CPU time advantage, those that are solved faster with SBCs often have a marked CPU time advantage (or, if not run to completion, a noticeable optimality gap or total/unexplored nodes ratio advantage). For MILPs and Narrowing1, for example (see Table 6), the cumulated CPU time advantage in favour of the original problem is 275 s, whereas that in favour of the SBC narrowing is 9861s. The trend seems to be that the beneficial effect of SBCs is mainly felt for medium to large-sized instances with long BB runs. Even though the optimal solution is often found later on in the BB run when solving SBC narrowings, the BB tree explorations are in general shorter. For those instances not solved to optimality, the ratios of total/unexplored nodes at termination are often larger (fewer unexplored nodes) and the open optimality gaps often smaller. This is what promtped us to run a second round of tests with no time limit for some selected difficult instances (see Table 11), on which these effects are even more remarkable.

Table 6 refers to MILPs (MIPLib3 and MIPLib2003), Table 7 refers to NLPs (GlobalLib) and Table 9 refers to MINLPs (MINLPLib). All tables have the same core structure recording the following indicators at termination:

1. incumbent value $\left(f^{*}\right)$

2. seconds of user CPU time $(C P U)$

3. open gap (gap - we use the CPLEX definition $\left(\frac{100\left|f^{*}-\bar{f}\right|}{\left|f^{*}+10^{-10}\right|}\right) \%$, where $f^{*}$ is the objective function value of the incumbent and $\bar{f}$ is the best overall lower bound)

4. total nodes (nodes)

5. unexplored nodes (tree)

for the original problem and each SBC narrowing. The last column (R.t.) contains the reformulation time expressed as seconds of user CPU time taken to reformulate the instance (this refers to Narrowing 1; the values for Narrowing 2 are practically identical, the bottleneck being the computation of the group structure by nauty). Tables 7 and 9 also have a column (Slv) which indicates the solver name: "C" stands for CouEnNE [4], and "B" for BARON [47]. Although COUENNE was our NLP/MINLP global solver of choice, because of its relatively young age it still shows some rough spots, which sometimes hamper the solution process. COUENNE failed on all instances whose results in the table are marked BARON. NLP and MINLP instances where both solvers failed are recorded in Tables 8 and 10: all these are well known to be difficult instances, and most of them are very large in size. We remark that for many of them the reason for failure was the absence of meaningful variable ranges, which makes the construction of the lower bounding problem inherently difficult. In all tables, data marked in boldface signals an advantage: in general, lower values for incumbent, CPU times, open gap, total and unexplored nodes at termination are considered 
an advantage. However, for those instances not solved to optimality within the $2 \mathrm{~h}$ CPU time limit, the higher values of the ratios total/unexplored nodes marks an advantage (meaning that more of the tree has been explored in the allotted time).

\begin{tabular}{|c|c|c|c|c|c|c|c|c|c|c|}
\hline \multirow[b]{2}{*}{ Instance } & \multicolumn{3}{|c|}{ Original problem } & \multicolumn{3}{|c|}{ Narrowing1 } & \multicolumn{3}{|c|}{ Narrowing2 } & \multirow[b]{2}{*}{ R.t. } \\
\hline & $C P U$ & $\begin{array}{c}f^{*} \\
g a p\end{array}$ & $\begin{array}{c}\begin{array}{c}\text { nodes } \\
\text { tree }\end{array} \\
\text { todes }\end{array}$ & $C P U$ & $\begin{array}{c}f^{*} \\
g a p\end{array}$ & $\begin{array}{c}\text { nodes } \\
\text { tree }\end{array}$ & $C P U$ & $\begin{array}{c}f^{*} \\
g a p\end{array}$ & $\begin{array}{c}\text { nodes } \\
\text { tree }\end{array}$ & \\
\hline \multicolumn{11}{|l|}{$\begin{array}{l}\text { MIPLib3 } \\
\end{array}$} \\
\hline & & 340160 & $\overline{0}$ & & 340160 & $\overline{0}$ & & 340160 & $\overline{0}$ & \\
\hline air03 & 1.14 & $0 \%$ & 0 & 1.10 & $0 \%$ & 0 & 0.98 & $0 \%$ & 0 & 161.94 \\
\hline & & $7.58 \mathrm{e}+6$ & 93340 & & $7.58 \mathrm{e}+6$ & 93340 & & $7.58 \mathrm{e}+6$ & 93340 & \\
\hline arki001 & 114.31 & $0 \%$ & 0 & 125.03 & $0 \%$ & 0 & 108.5 & $0 \%$ & 0 & 78.56 \\
\hline & & 7.59 & 957 & & 7.59 & 969 & & 7.59 & 967 & \\
\hline blend2 & 0.94 & $0 \%$ & 0 & 0.87 & $0 \%$ & 0 & 0.91 & $0 \%$ & 0 & 1.43 \\
\hline & & 0 & 0 & & 0 & 153 & & 0 & 153 & \\
\hline enigma & 0.00 & $0 \%$ & 0 & 0.05 & $0 \%$ & 0 & 0.05 & $0 \%$ & 0 & 1.22 \\
\hline & & $4.05 e+5$ & 71 & & $4.05 \mathrm{e}+5$ & 81 & & $4.05 e+5$ & 81 & \\
\hline fiber & 0.26 & $0 \%$ & 0 & 0.27 & $0 \%$ & 0 & 0.26 & $0 \%$ & 0 & 4.96 \\
\hline gen & 0.4 & $\begin{array}{c}1.12 \mathrm{e}+5 \\
0 \%\end{array}$ & $\begin{array}{l}0 \\
0\end{array}$ & 0.04 & $1.12 \mathrm{e}+5$ & $\begin{array}{l}0 \\
0\end{array}$ & 0.04 & $\begin{array}{c}1.12 \mathrm{e}+5 \\
0 \%\end{array}$ & $\begin{array}{l}0 \\
0\end{array}$ & 257 \\
\hline gent & 0.07 & $1.18 \mathrm{e}+4$ & 2405600 & 0.04 & $1.18 \mathrm{e}+4$ & 2558400 & 0.04 & $1.18 e+4$ & 2558400 & \\
\hline $\operatorname{mas} 74$ & 529 & $0 \%$ & 0 & 466 & $0 \%$ & 0 & 426 & $0 \%$ & 0 & 1.41 \\
\hline & & $4 \mathrm{e}+4$ & 305500 & & $4 e+4$ & 305500 & & $4 e+4$ & 305500 & \\
\hline $\operatorname{mas} 76$ & 43.42 & $0 \%$ & 0 & 43.62 & $0 \%$ & 0 & 41.97 & $0 \%$ & 0 & 1.41 \\
\hline & & 3360 & 160 & & 3360 & 656 & & 3360 & 700 & \\
\hline $\mathrm{misc} 03$ & 0.30 & $0 \%$ & 0 & 0.29 & $0 \%$ & 0 & 1.07 & $0 \%$ & 0 & 1.35 \\
\hline & & 0 & 17 & & 0 & 17 & & 0 & 17 & \\
\hline misc06 & 0.13 & $0 \%$ & 0 & 0.13 & $0 \%$ & 0 & 0.13 & $0 \%$ & 0 & 11.63 \\
\hline miscol & 18.41 & $\begin{array}{c}2810 \\
0 \%\end{array}$ & $\begin{array}{c}16211 \\
0\end{array}$ & 12.72 & $\begin{array}{c}2810 \\
0 \%\end{array}$ & $\begin{array}{c}12317 \\
0\end{array}$ & 21.28 & $\begin{array}{l}2810 \\
0 \%\end{array}$ & $\begin{array}{c}20395 \\
0\end{array}$ & 157 \\
\hline T150 & 10.71 & 115155 & 0 & & 115155 & 0 & & 115155 & 0 & \\
\hline mitre & 0.83 & $0 \%$ & 0 & 0.80 & $0 \%$ & 0 & 0.82 & $0 \%$ & 0 & 1304.41 \\
\hline & & -563.732 & 156803 & & -563.846 & 136200 & & & & \\
\hline mkc & 7200 & $0.17 \%$ & 75392 & 7200 & $0.15 \%$ & 36654 & - & - & & 2712.33 \\
\hline & & -41 & 8629594 & & -41 & 7852302 & & -41 & 7852302 & \\
\hline noswot & 7200 & $4.88 \%$ & 1581600 & 7200 & $3.56 \%$ & 817466 & 7200 & $3.56 \%$ & 817466 & 1.27 \\
\hline & & 7615 & 103 & & 7615 & 295 & & & & \\
\hline p0201 & 0.24 & $0 \%$ & 0 & 0.35 & $0 \%$ & 0 & - & - & - & 3.44 \\
\hline & & 3124 & 11 & & 3124 & 11 & & 3124 & 11 & \\
\hline p2756 & 0.40 & $0 \%$ & 0 & 0.39 & $0 \%$ & 0 & 0.37 & $0 \%$ & 0 & 25.61 \\
\hline giu & 45.01 & $\begin{array}{c}-132.87 \\
0 \%\end{array}$ & $\begin{array}{c}8375 \\
0\end{array}$ & 36.38 & $\begin{array}{c}-132.87 \\
0 \%\end{array}$ & $\begin{array}{c}\mathbf{5 5 0 0} \\
0\end{array}$ & - & - & - & 6.22 \\
\hline & & 82.2 & 561 & & 82.2 & 555 & & 82.2 & 505 & \\
\hline rgn & 0.13 & $0 \%$ & 0 & 0.15 & $0 \%$ & 0 & 0.12 & $0 \%$ & 0 & 1.36 \\
\hline & & 1077.21 & 5800 & & 1077.56 & 6700 & & 1077.56 & 17700 & \\
\hline rout & 11.99 & $0 \%$ & 0 & 15.28 & $0 \%$ & 0 & 53.86 & $0 \%$ & 0 & 2.39 \\
\hline & & 423 & 103097 & & 423 & 101576 & & 423 & 105481 & \\
\hline seymour & 7200 & $1.58 \%$ & 84949 & 7200 & $1.58 \%$ & 83701 & 7200 & $1.57 \%$ & 86941 & 5.95 \\
\hline & & 18 & 1582 & & 18 & 637 & & & & \\
\hline stein27 & 0.27 & $0 \%$ & 0 & 0.07 & $0 \%$ & & - & - & - & 1.30 \\
\hline & & 492.45 & 215100 & & 486.18 & 194400 & & 484.09 & 196200 & \\
\hline $\operatorname{swath}^{a}$ & 1534 & $14.60 \%$ & 200293 & 1773 & $13.83 \%$ & 165017 & 1550 & $17.55 \%$ & 182289 & 325.10 \\
\hline \multicolumn{11}{|c|}{ MIILLib2003 \MIPLib3 } \\
\hline & & $1.4 \mathrm{e}+9$ & 2240022 & & $1.2 \mathrm{e}+9$ & 392600 & & $1.2 \mathrm{e}+9$ & 392600 & \\
\hline glass4 & 7200 & $21.43 \%$ & 944291 & 1180.69 & $0 \%$ & 0 & 1186.57 & $0 \%$ & 0 & 1.62 \\
\hline & & -21718 & 543 & & -21718 & 588 & & -21718 & 588 & \\
\hline mzzv11 & 112.87 & $0 \%$ & 0 & 129.35 & $0 \%$ & 0 & 161 & $0 \%$ & 0 & 213.76 \\
\hline & & -20540 & 237 & & -20540 & 223 & & -20540 & 223 & \\
\hline $\mathrm{mzzv} 42 \mathrm{z}$ & 40.94 & $0 \%$ & 0 & 46.42 & $0 \%$ & 0 & 59.76 & $0 \%$ & 0 & 244.76 \\
\hline & & -16 & 0 & & -16 & 0 & & -16 & 0 & \\
\hline opt1217 & 0.09 & $0 \%$ & 0 & 0.09 & $0 \%$ & 0 & 0.10 & $0 \%$ & 0 & 1.37 \\
\hline & no & -19 & 12936 & & $\begin{array}{l}-19 \\
-19\end{array}$ & 14050 & 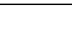 & 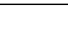 & 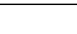 & (0) \\
\hline protfold & 7200 & $\frac{90.71 \%}{7.64 \mathrm{e}+5}$ & $\begin{array}{c}11563 \\
3576200\end{array}$ & 7200 & $\frac{91.58 \%}{7.64+5}$ & $\begin{array}{l}12760 \\
973700\end{array}$ & - & $7.64 \mathrm{e}+5$ & 973700 & 592.14 \\
\hline timtab1 & 5317.42 & $0 \%$ & 0 & 1554.20 & $0 \%$ & 0 & 1555.74 & $0 \%$ & 0 & 1.37 \\
\hline & & $7.12 e+6$ & 1115428 & & $7.14 \mathrm{e}+6$ & 1002113 & & $1.14 \mathrm{e}+6$ & 959893 & \\
\hline timtab2 & 7200 & $31.74 \%$ & 745016 & 7200 & $33.23 \%$ & 647515 & 7200 & $33.32 \%$ & 619402 & 1.38 \\
\hline
\end{tabular}

$a$ Termination on out of memory.

Table 6 MILP results (MIPLib3 and MIPLib2003 solved by CPLEX 11). Lower values are best in general; in instances not solved to optimality $(\mathrm{CPU}=7200)$, higher ratios nodes/tree are best. Values marked '-' denote Narrowing2=Narrowing1.

Some of the results for MILPs are very encouraging: the glass4 instance, for example, is known to be a hard one: [24] reports solving this instance using XPRESS 2006B in 7 hours on a 2 processor Xeon system with the following settings: no cuts, best first node selection, 
heavy strong branching, and variable selection based on up/down pseudocosts. Although it is hard to compare with our results, what with the solver, hardware and version date difference, solving it in less than 20 minutes on a default configuration is worthy of note; even more so considering that the symmetry group is only $C_{2}$. The timtab1 instance solution time is reduced to less than a third by adjoining the SBCs. It is interesting that arki001, mas76 and p2756 have the same number of nodes to completion but different CPU times. The CPLEX output $\log$ files of original/reformulated instances being equal for all but the partial CPU times, the only cause of this difference lies in the LP being solved at each node: although most of the times an LP with more constraints takes more time to solve, CPLEX employs several preprocessing techniques which might exploit the SBCs present in the reformulation (but absent in the original formulation) to yield the observed improvements.

On average, with a $2 \mathrm{~h}$ user CPU time limit, it is slightly more advantageous to solve an SBC narrowing than the original problem. We reported total user CPU time, number of times the solution yielded best optima in the set (Best), and total number of BB nodes. The total closed gap averaged over original problem and Narrowing1 and Narrowing2 reformulations is $22661.35 \%$ with a standard deviation of 0.14 , which effectively means that within the $2 \mathrm{~h}$ CPU time limit, symmetry breaking had no effect with respect to the closed gap (without the $2 \mathrm{~h}$ limit the story is different, see Table 11). It appears evident that, on average, breaking symmetry is beneficial when using BB-type solution algorithms.

The results referring to the second round of tests, involving selected (difficult) instances solved without the $2 \mathrm{~h}$ user CPU time limit, are in Table 11 . As before, data marked in boldface signals an advantage. The most meaningful indicators at termination are:

1. the objective function value of the incumbent (the lower the better);

2. the open gap (the lower the better);

3. the amount of explored nodes per second, i.e. $\frac{\text { nodes }}{\text { tree } \times C P U}$ (the higher the better).

With extended CPU time limits, Narrowing2 provides a significant computational advantage over the original problem, and a slight advantage over Narrowing1.

We remark also that results worthy of note were obtained on the protfold (open gap reduced by almost half) and seymour (given the problem structure, even a minor reduction in open gap is impressive) MILP instances.

It appears that adding SBCs sometimes has an adverse effect (albeit slighter than the beneficial observed effect). This occurrence may be explained by any one of the following facts: (a) SBCs have an element of arbitrary user choice in them, e.g. the natural variable index order 1,2,3,... (constraints enforcing other orders would also be valid); (b) SBCs may change branching decisions; (c) best choices for breaking symmetries may change during the BB tree exploration, locally to each node (it might be advantageous to change narrowing at select nodes rather than just at the root node). These issues will hopefully be addressed in future works (in particular, it might be a good idea to employ orbital branching $[43,44]$ instead of a static narrowing as a symmetry-breaking device).

\section{Conclusion}

This paper discusses methods for automatically exploiting symmetries in MILPs, nonconvex NLPs and MINLPs. We construct the formulation group, then derive static SymmetryBreaking Constraints from its generators, and finally reformulate the given problem to a narrowing where some of the symmetric solutions are likely to be infeasible. The reformulated problem can then be solved by standard Branch-and-Bound solvers such as CPLEX 


\begin{tabular}{|c|c|c|c|c|c|c|c|c|c|c|c|}
\hline \multirow[b]{2}{*}{ Instance } & \multirow[b]{2}{*}{$S l v$} & \multicolumn{3}{|c|}{ Original problem } & \multicolumn{3}{|c|}{ Narrowing1 } & \multicolumn{3}{|c|}{ Narrowing2 } & \multirow[b]{2}{*}{ R.t. } \\
\hline & & $C P U$ & $\begin{array}{l}f^{*} \\
\text { gap }\end{array}$ & $\begin{array}{c}\begin{array}{c}\text { nodes } \\
\text { tree }\end{array} \\
\end{array}$ & $C P U$ & $\begin{array}{l}f^{*} \\
\text { gap }\end{array}$ & $\begin{array}{c}\text { nodes } \\
\text { tree }\end{array}$ & $C P U$ & $\begin{array}{c}f^{*} \\
\text { gap }\end{array}$ & $\begin{array}{c}\text { nodes } \\
\text { tree }\end{array}$ & \\
\hline ex14_1_5 & C & 0.018 & $0 \%$ & $\begin{array}{l}0 \\
0\end{array}$ & 0.013 & $\begin{array}{l}0 \\
0 \%\end{array}$ & $\begin{array}{l}0 \\
0\end{array}$ & 0.020 & $\begin{array}{c}0 \\
0 \%\end{array}$ & $\begin{array}{l}0 \\
0\end{array}$ & 1.44 \\
\hline ex2_1_3 & C & 0.013 & $\begin{array}{l}-15 \\
0 \%\end{array}$ & $\begin{array}{l}0 \\
0\end{array}$ & 0.010 & $\begin{array}{l}-15 \\
0 \%\end{array}$ & $\begin{array}{l}0 \\
0\end{array}$ & 0.018 & $\begin{array}{l}-15 \\
0 \%\end{array}$ & $\begin{array}{l}0 \\
0\end{array}$ & 1.41 \\
\hline ex5 2.5 & $\mathrm{C}$ & 7200 & $\begin{array}{l}-3500 \\
0.32 \%\end{array}$ & $\begin{array}{c}2040274 \\
503850\end{array}$ & 7200 & $\begin{array}{l}-3500 \\
0.27 \%\end{array}$ & $\begin{array}{l}63595 \\
18733\end{array}$ & 7200 & $\begin{array}{l}-3500 \\
\mathbf{0 . 2 2} \%\end{array}$ & $\begin{array}{l}1580366 \\
402117\end{array}$ & 1.40 \\
\hline & & & $-2.02 \mathrm{e}-2$ & 14226 & & $-2.02 \mathrm{e}-02$ & 10027 & & $-2.02 \mathrm{e}-02$ & 10027 & \\
\hline ex6_1_1 & $\mathrm{C}$ & 61 & $0 \%$ & 0 & 37 & $0 \%$ & 0 & 45 & $0 \%$ & 0 & 1.43 \\
\hline & & & $-3.53 \mathrm{e}-01$ & 13660 & & $-3.53 \mathrm{e}-01$ & 2659 & & $-3.53 \mathrm{e}-01$ & 2659 & \\
\hline ex6_1_3 & $\mathrm{C}$ & 135 & $\frac{0 \%}{-3.052}$ & $\frac{0}{45200}$ & 97 & $\frac{0 \%}{-3.052}$ & $\frac{0}{88300}$ & 1111 & $\frac{0 \%}{-3.052}$ & $\frac{0}{81700}$ & 1.40 \\
\hline ex6_2_-10 ${ }^{a}$ & C & 4754 & $0.37 \%$ & 19397 & 7200 & $0.01 \%$ & 16228 & 7200 & $0.014 \%$ & 15898 & 1.40 \\
\hline ex6_2_12 & $\mathrm{C}$ & 205 & $\begin{array}{c}2.89 \mathrm{e}-01 \\
0 \%\end{array}$ & $\begin{array}{c}15827 \\
0\end{array}$ & 85 & $\begin{array}{c}2.89 \mathrm{e}-01 \\
0 \%\end{array}$ & $\begin{array}{c}3477 \\
0\end{array}$ & 85 & $\begin{array}{c}2.89 \mathrm{e}-01 \\
0 \%\end{array}$ & $\begin{array}{c}3477 \\
0\end{array}$ & 1.41 \\
\hline ex6_2_13 & $\mathrm{C}$ & 7200 & $\begin{array}{c}-2.16 e-01 \\
0.09 \%\end{array}$ & $\begin{array}{l}65461 \\
27773\end{array}$ & 7200 & $\begin{array}{l}-2.16 \mathrm{e}-01 \\
0.03 \%\end{array}$ & $\begin{array}{l}77569 \\
29202\end{array}$ & 7200 & $\begin{array}{l}-2.16 \mathrm{e}-01 \\
03 \%\end{array}$ & $\begin{array}{l}75670 \\
28580\end{array}$ & $138-2$ \\
\hline exu-zoto & & & $-6.96 \mathrm{e}-01$ & 195 & & $-6.96 \mathrm{e}-01$ & 92 & 1200 & $-6.96 \mathrm{e}-01$ & 92 & 1.50 \\
\hline ex6_2_14 & $\mathrm{C}$ & 29 & $0 \%$ & 0 & 19 & $0 \%$ & 0 & 17.8 & $0 \%$ & 0 & 1.37 \\
\hline & & & -70.75 & 43500 & & -70.75 & 68600 & & -70.75 & 68600 & \\
\hline ex6_2_-5 ${ }^{a}$ & $\mathrm{C}$ & 3017 & $6.85 \%$ & 18094 & 4920 & $0.90 \%$ & 27187 & 5240 & $0.90 \%$ & 27187 & 1.43 \\
\hline & & & -0.161 & 32500 & & -0.161 & 17500 & & -0.161 & 16600 & \\
\hline ex6_2_- $7^{a}$ & $\mathrm{C}$ & 1874 & $48.35 \%$ & 10900 & 1884 & $39.92 \%$ & 7894 & 1323 & $32.07 \%$ & 8008 & 1.42 \\
\hline 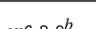 & & & $-3.51 \mathrm{e}-02$ & $287 \pi$ & & $-3.46 \mathrm{e}-02$ & 8522 & & $-3.46 e-02$ & 8522 & \\
\hline ex6____ $9^{D}$ & $\mathrm{C}$ & 699 & $\frac{0 \%}{5065}$ & 0 & 184 & $0 \%$ & 0 & 191 & $0 \%$ & 0 & 1.42 \\
\hline ex8_1_6 & $\mathrm{C}$ & 0.03 & $\begin{array}{c}-3.00 \\
0 \% \\
\end{array}$ & 0 & 0.03 & $\begin{array}{c}-3.003 \\
0 \%\end{array}$ & 0 & 0.046 & $\begin{array}{c}-3.003 \\
0 \%\end{array}$ & 0 & 1.36 \\
\hline ex8_3_. ${ }^{c}$ & B & 7200 & $\begin{array}{c}0 \\
-10\end{array}$ & $\begin{array}{l}20245 \\
12299\end{array}$ & 7200 & $\begin{array}{c}0 \\
-10\end{array}$ & $\begin{array}{l}14191 \\
8099\end{array}$ & - & - & - & 2.27 \\
\hline & & & -0.4123 & 9471 & & -0.4123 & 8004 & & & & \\
\hline ex8_3_2 & $\mathrm{B}$ & 7200 & $2325 \%$ & 6566 & 7200 & $2325 \%$ & 5445 & - & - & - & 2.05 \\
\hline ex8_3_3 & B & 7200 & $\begin{array}{l}-0.4100 \\
2393 \% \\
\end{array}$ & 5770 & 7200 & $2393 \%$ & $\begin{array}{r}7410 \\
5065\end{array}$ & - & - & - & 1.36 \\
\hline ex8_3_4 & в & 7200 & $\begin{array}{l}-3.58 \\
2695 \%\end{array}$ & $\begin{array}{l}6597 \\
4484\end{array}$ & 7200 & $\begin{array}{c}-3.58 \\
2695 \%\end{array}$ & $\begin{array}{l}4985 \\
3347\end{array}$ & - & - & - & 2.10 \\
\hline & & & -0.069 & 7843 & & -0.068 & 8470 & & & & \\
\hline ex8_3_5 & B & 7200 & $14371 \%$ & 4727 & 7200 & $14434 \%$ & 5597 & - & - & - & 2.07 \\
\hline ex8_3_11 ${ }^{c}$ & B & 7200 & $\begin{array}{c}0 \\
-10\end{array}$ & $\begin{array}{l}15197 \\
8393\end{array}$ & 7200 & $\begin{array}{c}0 \\
-10\end{array}$ & $\begin{array}{l}21532 \\
13344\end{array}$ & . & - & - & 151 \\
\hline & & & $\frac{10}{0}$ & 21881 & & $\frac{-10}{0}$ & 20566 & & - & - & 1.01 \\
\hline ex8-3 $-12^{c}$ & B & 7200 & -10 & 12515 & 7200 & -10 & 13000 & - & - & - & 1.37 \\
\hline ex8_3_13 ${ }^{c}$ & B & 7200 & $\begin{array}{c}0 \\
-10\end{array}$ & $\begin{array}{l}13662 \\
9015\end{array}$ & 7200 & $\begin{array}{c}0 \\
-10\end{array}$ & $\begin{array}{l}11038 \\
7179\end{array}$ & - & - & - & 1.91 \\
\hline ex8_4_6 & $\mathrm{C}$ & 0.08 & $\begin{array}{l}0.66 \\
0 \%\end{array}$ & $\begin{array}{l}0 \\
0\end{array}$ & 1.53 & $\begin{array}{c}0.66 \\
0 \%\end{array}$ & $\begin{array}{l}0 \\
0\end{array}$ & 0.13 & $\begin{array}{c}0.66 \\
0 \%\end{array}$ & $\begin{array}{l}0 \\
0\end{array}$ & 1.40 \\
\hline & & & -3.25 & 0 & & -3.25 & 0 & & -3.25 & 0 & \\
\hline ex9_1_10 & $\mathrm{C}$ & 4.68 & $0 \%$ & 0 & 9.3 & $0 \%$ & 0 & 9.3 & $0 \%$ & 0 & 1.77 \\
\hline $0 \times 918$ & $C$ & 47 & -3.25 & 0 & 93 & -3.25 & 0 & $933-3$ & -3.25 & 0 & $437>>$ \\
\hline ext-1_o & C & 4.1 & 99.99 & $\frac{0}{2}$ & 9.5 & 99.99 & $\frac{0}{2}$ & 9.0 & 99.99 & $\frac{0}{2}$ & 1.43 \\
\hline ex9_2_2 & $\mathrm{C}$ & 0.16 & $0 \%$ & 0 & 0.15 & $0 \%$ & 0 & 0.17 & $0 \%$ & 0 & 2.31 \\
\hline ex9_2_6 & C & 0.1 & $\begin{array}{l}-1 \\
0 \%\end{array}$ & $\begin{array}{l}0 \\
0\end{array}$ & 0.1 & $\begin{array}{l}-1 \\
0 \%\end{array}$ & $\begin{array}{l}0 \\
0\end{array}$ & - & - & - & 1.38 \\
\hline & & & -0.366 & 31655 & & -0.366 & 28973 & & -0.366 & 31117 & \\
\hline maxmin & B & 7200 & $157.55 \%$ & 20122 & 7200 & $157.38 \%$ & 18300 & 7200 & $156.87 \%$ & 19659 & 1.29 \\
\hline & & & -2.83 & 0 & & -2.83 & 0 & & -2.83 & 0 & \\
\hline st_e18 & $\mathrm{C}$ & 0.01 & $0 \%$ & 0 & 0.01 & $0 \%$ & 0 & 0.01 & $0 \%$ & 0 & 1.38 \\
\hline (et & $\mathrm{S}_{\mathrm{s}}$ & 0.03 & $\begin{array}{l}-5.065 \\
06\end{array}$ & 0 & 0.03 & -5.065 & 0 & 004 & -5.065 & 0 & 141 \\
\hline 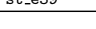 & $\mathrm{C}$ & 0.00 & -15 & 0 & $0.0 \mathrm{~S}$ & -15 & 0 & 0.04 & -15 & 0 & 1.41 \\
\hline st_fp3 & C & 0.015 & $0 \%$ & 0 & 0.015 & $0 \%$ & 0 & 0.017 & $0 \%$ & 0 & 2.02 \\
\hline st $r x^{\circ}$ & $\mathrm{C}$ & 103 & $\begin{array}{c}-120.15 \\
0 \%\end{array}$ & $\begin{array}{l}214 \\
0\end{array}$ & 95 & $\begin{array}{c}-120.15 \\
0 \%\end{array}$ & 208 & $933 \longrightarrow>$ & $\begin{array}{c}-120.15 \\
0 \%\end{array}$ & 208 & 144 \\
\hline & & & -28371.6 & 97 & & -28371.6 & 125 & & -28371.6 & 125 & \\
\hline turkey & $\mathrm{C}$ & 2749 & $0 \%$ & 0 & 3724 & $0 \%$ & 0 & 3831 & $0 \%$ & 0 & 7.24 \\
\hline
\end{tabular}

a $C P U<7200$ and gap $>0 \%$ because of COUENNE's segmentation fault during computation.

$b$ Some AMPL warnings might be the cause of the objective function value discrepancy (both were certified optimal by COUENNE).

$c$ When $f^{*}=0$ the open gap is (almost) ill defined, thus the value of the best LP bound is reported instead.

Table 7 NLP results (GlobalLib solved by COUENNE or BARON). Lower values are best in general; in instances not solved to optimality $(\mathrm{CPU}=7200)$, higher ratios nodes/tree are best. Values marked '-' denote Narrowing2=Narrowing1.

(for linear problems) and CouENNE (for nonlinear problems). We exhibit computational results validating the approach. 


\begin{tabular}{|c|c|c|c|c|c|c|c|}
\hline Instance & R.t. & & & Instance & R.t. & Instance & R.t. \\
\hline arki0002 & 82.36 & Instance & R.t. & elec25 & 5.32 & ex8_3_6 & 1.45 \\
\hline $\operatorname{arki0003}$ & 10813 & $\operatorname{arki0012}$ & $\begin{array}{l}5400.03 \\
526854\end{array}$ & elec50 & 228.67 & ex8_3_7 & 1.62 \\
\hline arki0008 & 92.15 & arki0013 & $\begin{array}{l}5268.34 \\
5695.04\end{array}$ & ex8_2_1 & 1.43 & ex8_3_9 & 1.41 \\
\hline arki0009 & 401.10 & arki0014 & 5695.04 & ex8_2_2 & 21080 & ex8_3_10 & 1.28 \\
\hline arki0010 & 65.46 & arkivolo & $\begin{array}{r}142.34 \\
1308236\end{array}$ & ex8_2_4 & 1.64 & ex8_3_14 & 1.48 \\
\hline $\operatorname{arki0011}$ & 5715.02 & & 15082.50 & ex8_2_5 & 17172 & torsion50 & 6122 \\
\hline
\end{tabular}

Table 8 NLP instances where both COUENNE and BARON failed.

\begin{tabular}{|c|c|c|c|c|c|c|c|c|c|c|c|}
\hline \multirow[b]{2}{*}{ Instance } & \multirow[b]{2}{*}{$S l v$} & \multicolumn{3}{|c|}{ Original problem } & \multicolumn{3}{|c|}{ Narrowing1 } & \multicolumn{3}{|c|}{ Narrowing2 } & \multirow[b]{2}{*}{ R.t. } \\
\hline & & $C P U$ & $\begin{array}{l}f^{*} \\
g a p\end{array}$ & $\begin{array}{c}\begin{array}{c}\text { nodes } \\
\text { tree }\end{array} \\
\text { toded }\end{array}$ & $C P U$ & $\begin{array}{l}f^{*} \\
g a p\end{array}$ & $\begin{array}{c}\begin{array}{c}\text { nodes } \\
\text { tree }\end{array} \\
\text { thes }\end{array}$ & $C P U$ & $\begin{array}{l}f^{*} \\
g a p\end{array}$ & $\begin{array}{c}\begin{array}{c}\text { nodes } \\
\text { tree }\end{array} \\
\text { ofes }\end{array}$ & \\
\hline & & & $-1.14 \mathrm{e}+5$ & $\begin{array}{ll}106074 \\
\end{array}$ & & $-1.14 \mathrm{e}+5$ & 106074 & & $-1.14 \mathrm{e}+5$ & 101138 & \\
\hline cecil_13 & C & 6181 & $0 \%$ & 0 & 6248 & $0 \%$ & 0 & 7200 & $0 \%$ & $2084 \downarrow$ & 2.64 \\
\hline & & & 0.1916 & 341 & & 0.1916 & 326 & & 0.1916 & 87 & \\
\hline elf & B & 11.86 & $0 \%$ & 0 & 7.35 & $0 \%$ & 0 & 2.9 & $0 \%$ & 0 & 1.43 \\
\hline & & & 89.1 & 227 & & 89.1 & 109 & & 89.1 & 109 & \\
\hline gastrans & C & 9 & $0 \%$ & 0 & 5.2 & $0 \%$ & 0 & 5.7 & $0 \%$ & 0 & 1.39 \\
\hline raar & $C$ & 0,0 & 0 & 8 & 001 & $\begin{array}{c}0 \\
0 \%\end{array}$ & $\begin{array}{l}0 \\
0\end{array}$ & 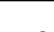 & 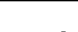 & 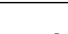 & 127 \\
\hline & & & 0 & 6 & 0.01 & $\frac{0}{0}$ & 21 & - & & & 1.27 \\
\hline gear2 & $\mathrm{C}$ & 0.34 & $0 \%$ & 0 & 0.51 & $0 \%$ & 0 & - & - & - & 1.38 \\
\hline gear3 & $\mathrm{C}$ & 0.14 & $0 \%$ & $\begin{array}{c}26 \\
0\end{array}$ & 0.19 & $0 \%$ & $\begin{array}{c}25 \\
0\end{array}$ & - & - & - & 1.30 \\
\hline & & & 1.968 & 3239 & & 1.968 & 1739 & & & & 1.000 \\
\hline gear4 & B & 0.62 & $0 \%$ & 0 & 0.48 & $0 \%$ & 0 & - & - & - & 1.28 \\
\hline & & & 13 & 0 & & 13 & 0 & & 13 & 0 & \\
\hline hmittelman & $\mathrm{C}$ & 0.16 & $0 \%$ & 0 & 0.18 & $0 \%$ & 0 & 0.20 & $0 \%$ & 0 & 1.25 \\
\hline & & & 4492.48 & 9106 & & 4493.5 & 10146 & & 4457.55 & 3254 & \\
\hline lop97icx & B & 7200 & $40.65 \%$ & 5537 & 7200 & $39.9 \%$ & 6296 & 7200 & $40.23 \%$ & 2026 & 24.96 \\
\hline & & & $\begin{array}{c}-43.13 \\
0 \%\end{array}$ & $\begin{array}{l}0 \\
0\end{array}$ & & $\begin{array}{c}-43.13 \\
0 \%\end{array}$ & $\begin{array}{l}0 \\
0\end{array}$ & & $\begin{array}{c}-43.13 \\
0 \%\end{array}$ & $\begin{array}{l}0 \\
0\end{array}$ & \\
\hline nvs09 & $\mathrm{C}$ & 5.1 & $\frac{0 \%}{558}$ & 0 & 2.4 & $0 \%$ & 0 & 1.7 & $0 \%$ & 0 & 1.24 \\
\hline risk2 $\mathrm{b}^{a}$ & C & 13.26 & $\begin{array}{c}55.87 \\
0 \%\end{array}$ & 0 & 14.77 & 55.87 & $\begin{array}{l}0 \\
0\end{array}$ & 14.28 & $\begin{array}{l}55.87 \\
0 \%\end{array}$ & $\begin{array}{l}0 \\
0\end{array}$ & 2.48 \\
\hline & & & $1.5 e+5$ & 3316 & & $1.5 e+5$ & 1775 & & $1.5 \mathrm{e}+5$ & 9509 & \\
\hline synheat & B & 127 & $0 \%$ & 0 & 92 & $0 \%$ & 0 & 566 & $0 \%$ & 0 & 1.27 \\
\hline
\end{tabular}

$a$ This instance is unbounded [33], so the objective function value is not a meaningful indicator.

Table 9 MINLP results (MINLPLib solved by COUENNE or BARON). Lower values are best in general; in instances not solved to optimality $(\mathrm{CPU}=7200)$, higher ratios nodes/tree are best. Values marked '-' denote Narrowing2=Narrowing1.

\begin{tabular}{|c|c|c|c|c|c|c|c|}
\hline Instance & R.t. & Instance & R.t. & Instance & R.t. & Instance & R.t. \\
\hline deb7 & 26.0 & nuclear24 & 15.22 & nuclearvc & 1.88 & product2 & 292.39 \\
\hline deb8 & 26.1 & nuclear25 & 19.46 & nuclearvd & 2.26 & super1 & 10.12 \\
\hline deb9 & 25.9 & nuclear 49 & 457.88 & nuclearve & 2.03 & super2 & 10.60 \\
\hline lop97ic & 202.85 & nuclearva & 1.83 & nuclearvf & 2.03 & super3 & 10.15 \\
\hline nuclear 14 & 15.49 & nuclearvb & 1.73 & product & 13.88 & super $3 t$ & 6.09 \\
\hline
\end{tabular}

Table 10 MINLP instances where both COUENNE and BARON failed (the deb instances are reported infeasible).

Symmetry-Breaking Constraints practically help finding exact optima by Branch-andBound algorithms: in general, the more symmetry-breaking constraints we adjoin to the original formulation, the fewer nodes we might hope the BB search tree will have. Furthermore, these constraints are generated by the nontrivial orbits of the formulation group action on the set of variable indices. Therefore, in general, the larger the formulation group, the better. Since different exact reformulations of the same problem often yield different formulation groups (all of which are subgroups of the solution group associated to the problem), a very interesting question for future research is that of looking for the exact reformulation maximizing the number (and length) of nontrivial orbits. It must be said, however, that our symmetry-breaking constraints are rather general-purpose, hence they undergo the usual trade-off between generality and efficiency. This suggests that breaking symmetries at the modelling level (static symmetry breaking) should also be complemented by breaking sym- 


\begin{tabular}{|c|c|c|c|c|c|c|c|c|c|c|c|}
\hline \multirow[b]{2}{*}{ Instance } & \multirow[b]{2}{*}{$S l v$} & \multicolumn{3}{|c|}{ Original problem } & \multicolumn{3}{|c|}{ Narrowing1 } & \multicolumn{3}{|c|}{ Narrowing2 } & \multirow[b]{2}{*}{ R.t. } \\
\hline & & $C P U$ & $\begin{array}{l}f^{*} \\
\text { gap }\end{array}$ & $\begin{array}{c}\text { nodes } \\
\text { tree }\end{array}$ & $C P U$ & $\begin{array}{l}f^{*} \\
\text { gap }\end{array}$ & $\begin{array}{c}\text { nodes } \\
\text { tree }\end{array}$ & $C P U$ & $\begin{array}{l}f^{*} \\
\text { gap }\end{array}$ & $\begin{array}{c}\text { nodes } \\
\text { tree }\end{array}$ & \\
\hline \multicolumn{12}{|l|}{ MILPLib(s) } \\
\hline & & & -563.846 & 7945500 & & -563.846 & 2104500 & & & & \\
\hline $\mathrm{mkc}^{a}$ & & 146850 & $0.13 \%$ & 1479080 & 133924 & $0.13 \%$ & 1449867 & - & - & - & 2712.33 \\
\hline protfold $d^{b}$ & & 300000 & $\begin{array}{c}-26 \\
30.51 \%\end{array}$ & $\begin{array}{l}592000 \\
458813\end{array}$ & 300000 & $\begin{array}{c}-29 \\
16.54 \%\end{array}$ & $\begin{array}{l}536100 \\
353823\end{array}$ & - & - & & 592.14 \\
\hline & & & 423 & 3992700 & & 423 & 4343500 & & 423 & 3960700 & \\
\hline seymour $^{a}$ & & 262817 & $0.9 \%$ & 3026077 & 283311 & $0.83 \%$ & 3038821 & 233643 & $1.0 \%$ & 3064665 & 5.95 \\
\hline \multicolumn{12}{|l|}{ GlobalLib } \\
\hline & & & -3500 & 5452500 & & -3500 & 7373700 & & -3500 & 4425400 & \\
\hline ex5_2_.5 ${ }^{a}$ & $\mathrm{C}$ & 19805 & $28.14 \%$ & 1259853 & 82320 & $18.5 \%$ & 747262 & 18151 & $17.41 \%$ & 1076927 & 1.40 \\
\hline $\operatorname{maxmin} a$ & B & 58643 & $\begin{array}{l}-0.366 \\
145 \%\end{array}$ & 237100 & & -0.366 & $\begin{array}{l}238000 \\
150355\end{array}$ & - & - & - & 20 \\
\hline \multicolumn{12}{|l|}{ MINLPLib } \\
\hline & & & 4391.1 & 44858 & & 4493.5 & 43948 & & 4412.9 & 23416 & \\
\hline lop97icx ${ }^{a}$ & B & 26903 & $38.2 \%$ & 27824 & 30772 & $39.14 \%$ & 27189 & 42926 & $37.97 \%$ & 14708 & 24.96 \\
\hline
\end{tabular}

$a$ Termination on out of memory.

$b$ Termination after 5000 minutes

Table 11 Some results without the $2 \mathrm{~h}$ CPU time limit. Lower values are best in general; in instances not solved to optimality, higher ratios nodes/(tree $\times C P U)$ are best.

metries at the branching level of the BB algorithm (dynamic symmetry breaking). This will make the object of further investigations.

The tabulation of the formulation groups for all instances in the best known mathematical programming libraries suggests that although symmetry is not all-encompassing, it is nonetheless pervasive enough to merit more attention than is currently attributed to it by the mathematical programming community. Current efforts are limited to Mixed-Integer Linear and Semidefinite Programming only (this is the first work reaching into Mixed-Integer Nonlinear Programming) and often assume prior knowledge of (subgroups of) the solution group. If efficient symmetry detection and breaking devices are to make their way into mainstream MINLP solvers, more techniques are needed to address the issues arising in treating symmetry in mathematical programming.

\section{Acknowledgements}

I wish to thank François Margot for many useful discussions and suggestions, and for carefully checking the theoretical part of this paper, and two anonymous referees for insightful comments. Financial support by ANR under grant 07-JCJC-0151, by EU under grant NEST "Morphex" and by the Digiteo RMNCCO Chair is gratefully acknowledged.

\section{References}

1. F.A. Al-Khayyal and H.D. Sherali. On finitely terminating branch-and-bound algorithms for some global optimization problems. SIAM Journal of Optimization, 10(4):1049-1057, 2000.

2. L. Babai. Automorphism groups, isomorphism, reconstruction. In R. Graham, M. Grötschel, and L. Lovász, editors, Handbook of Combinatorics, vol. 2, pages 1447-1540. MIT Press, Cambridge, MA, 1996.

3. C. Bauer, A. Frink, and R. Kreckel. Introduction to the GiNaC framework for symbolic computation within the C++ programming language. Journal of Symbolic Computation, 33(1):1-12, 2002.

4. P. Belotti, J. Lee, L. Liberti, F. Margot, and A. Wächter. Branching and bounds tightening techniques for non-convex MINLP. Optimization Methods and Software, 24(4):597-634, 2009. 
5. T. Berthold and M. Pfetsch. Detecting orbitopal symmetries. In B. Fleischmann, K.-H. Borgwardt, R. Klein, and A. Tuma, editors, Operations Research Proceedings 2008, pages 433-438, Berlin, 2009. Springer.

6. R. Bixby, S. Ceria, C. McZeal, and M. Savelsbergh. An updated mixed integer programming library: Miplib 3. Technical Report TR98-03, Rice University, 1998.

7. K. Booth and C. Colbourn. Problems polynomially equivalent to graph isomorphism. Technical Report CS-77-04, University of Waterloo, 1979

8. M. Boulle. Compact mathematical formulation for graph partitioning. Optimization and Engineering, 5:315-333, 2004.

9. M. Bruglieri and L. Liberti. Optimal running and planning of a biomass-based energy production process. Energy Policy, 36:2430-2438, 2008.

10. M. Bussieck. Globallib - a collection of nonlinear programming problems, 2004. (http://www.gamsworld.org/global/globallib.htm).

11. M. Bussieck, A. Drud, and A. Meeraus. MINLPLib - A collection of test models for mixed-integer nonlinear programming. INFORMS Journal on Computing, 15(1), 2003.

12. G. Butler. Fundamental Algorithms for Permutation Groups, volume 559 of LNCS. Springer, 1991.

13. D. Cohen, P. Jeavons, C. Jefferson, K. Petrie, and B. Smith. Symmetry definitions for constraint satisfaction problems. In P. van Beek, editor, CP, volume 3709 of $L N C S$. Springer, 2005.

14. J.S. Cohen. Computer Algebra and Symbolic Computation: Mathematical Methods. AK Peters, Natick, Massachusetts, 2000

15. J.S. Cohen. Computer Algebra and Symbolic Computation: Elementary Algorithms. AK Peters, Natick, Massachusetts, 2002

16. Y. Faenza and V. Kaibel. Extended formulations for packing and partitioning orbitopes. Mathematics of Operations Research, 34(3):686-697, 2009.

17. M. Fischetti and D. Salvagnin. A local dominance procedure for mixed-integer linear programming. Technical report, ARRIVAL project, 2007.

18. R. Fourer and D. Gay. The AMPL Book. Duxbury Press, Pacific Grove, 2002.

19. E.J. Friedman. Fundamental domains for integer programs with symmetries. In A. Dress, Y. Xu, and B. Zhu, editors, COCOA Proceedings, volume 4616 of LNCS, pages 146-153. Springer, 2007.

20. The GAP Group. GAP - Groups, Algorithms, and Programming, Version 4.4.10, 2007.

21. M. Hall. Theory of Groups. Chelsea Publishing Company, New York, 2nd edition, 1976.

22. ILOG. ILOG CPLEX 11.0 User's Manual. ILOG S.A., Gentilly, France, 2008.

23. V. Kaibel and M. Pfetsch. Packing and partitioning orbitopes. Mathematical Programming, 114(1):1-36, 2008.

24. R. Laundy, M. Perregaard, G. Tavares, H. Tipi, and A. Vazacopoulos. Solving hard mixed-integer programming problems with Xpress-MP: A MIPLIB 2003 case study. INFORMS Journal on Computing, 21(2):304-313, 2009.

25. J. Lee and F. Margot. On a binary-encoded ILP coloring formulation. INFORMS Journal on Computing, 19(3):406-415, 2007.

26. S. Leyffer. MacMINLP - AMPL collection of mixed integer nonlinear programs, 2000. (http://www.mcs.anl.gov/ leyffer/macminlp/)

27. L. Liberti. Framework for symbolic computation in $\mathrm{C}++$ using $n$-ary trees. Technical report, CPSE, Imperial College London, 2001.

28. L. Liberti. Writing global optimization software. In L. Liberti and N. Maculan, editors, Global Optimization: from Theory to Implementation, pages 211-262. Springer, Berlin, 2006.

29. L. Liberti. Automatic generation of symmetry-breaking constraints. In B. Yang, D.-Z. Du, and C.A. Wang, editors, COCOA Proceedings, volume 5165 of LNCS, pages 328-338, Berlin, 2008. Springer.

30. L. Liberti. Reformulations in mathematical programming: Symmetry. Technical Report 2165, Optimization Online, 2008

31. L. Liberti. Reformulations in mathematical programming: Definitions and systematics. RAIRO-RO, 43(1):55-86, 2009

32. L. Liberti, S. Cafieri, and F. Tarissan. Reformulations in mathematical programming: A computational approach. In A. Abraham, A.-E. Hassanien, P. Siarry, and A. Engelbrecht, editors, Foundations of Computational Intelligence Vol. 3, number 203 in Studies in Computational Intelligence, pages 153-234. Springer, Berlin, 2009

33. L. Liberti, N. Mladenović, and G. Nannicini. A good recipe for solving MINLPs. In V. Maniezzo, T. Stützle, and S. Voß, editors, Hybridizing metaheuristics and mathematical programming, volume 10 of Annals of Information Systems, pages 231-244, New York, 2009. Springer.

34. F. Margot. Pruning by isomorphism in branch-and-cut. Mathematical Programming, 94:71-90, 2002.

35. F. Margot. Exploiting orbits in symmetric ILP. Mathematical Programming B, 98:3-21, 2003.

36. F. Margot. Small covering designs by branch-and-cut. Mathematical Programming B, 94:207-220, 2003. 
37. F. Margot. Symmetric ILP: coloring and small integers. Discrete Optimization, 4:40-62, 2007.

38. F. Margot. Symmetry in integer linear programming. In M. Jünger, T. Liebling, D. Naddef, G. Nemhauser, W. Pulleyblank, G. Reinelt, G. Rinaldi, and L. Wolsey, editors, 50 Years of Integer Programming, pages 647-681. Springer, Berlin, 2010.

39. A. Martin, T. Achterberg, and T. Koch. Miplib 2003. Technical Report 05-28, ZIB, 2005.

40. B. McKay. Practical graph isomorphism. Congressus Numerantium, 30:45-87, 1981.

41. B. McKay. nauty User's Guide (Version 2.4). Computer Science Dept., Australian National University, 2007.

42. A. Neumaier. Complete search in continuous global optimization and constraint satisfaction. Acta Numerica, 13:271-369, 2004.

43. J. Ostrowski, J. Linderoth, F. Rossi, and S. Smriglio. Orbital branching. In M. Fischetti and D.P. Williamson, editors, IPCO, volume 4513 of LNCS, pages 104-118. Springer, 2007.

44. J. Ostrowski, J. Linderoth, F. Rossi, and S. Smriglio. Constraint orbital branching. In A. Lodi, A. Panconesi, and G. Rinaldi, editors, IPCO, volume 5035 of LNCS, pages 225-239. Springer, 2008.

45. A. Ramani and I. Markov. Automatically exploiting symmetries in constraint programming. In B. Faltings, A. Petcu, F. Fages, and F. Rossi, editors, Constraint Solving and Constraint Logic Programming, volume 3419 of $L N A I$, pages 98-112, Berlin, 2005. Springer.

46. K.H. Rosen, editor. Handbook of Discrete and Combinatorial Mathematics. CRC Press, New York, 2000.

47. N.V. Sahinidis and M. Tawarmalani. BARON 7.2.5: Global Optimization of Mixed-Integer Nonlinear Programs, User's Manual, 2005.

48. H. Schichl and A. Neumaier. Interval analysis on directed acyclic graphs for global optimization. Journal of Global Optimization, 33(4):541-562, 2005.

49. A. Seress. Permutation Group Algorithms. Cambridge University Press, Cambridge, 2003.

50. H. Sherali and C. Smith. Improving discrete model representations via symmetry considerations. Management Science, 47(10):1396-1407, 2001.

51. R. Uehara, S. Toda, and T. Nagoya. Graph isomorphism completeness for chordal bipartite graphs and strongly chordal graphs. Discrete Applied Mathematics, 145:479-482, 2005.

52. F. Vallentin. Symmetry in semidefinite programs. Technical Report 1702, Optimization Online, 2007.

53. H. Wielandt. Finite permutation groups. Academic Press, New York, 1964

54. L.A. Wolsey. Integer Programming. Wiley, New York, 1998.

55. W. Zhu. Unsolvability of some optimization problems. Applied Mathematics and Computation, 174:921-926, 2006. 
Online appendix: Instance size tables

\begin{tabular}{|c|c|c|c|c|}
\hline Instance & $n$ & Bin. & Int. & $m$ \\
\hline 10teams & 2025 & 1800 & 0 & 230 \\
\hline $\begin{array}{l}\text { airro3 } \\
\text { air04 }\end{array}$ & $\begin{array}{r}10757 \\
8904\end{array}$ & $\begin{array}{r}10757 \\
8904\end{array}$ & $\begin{array}{l}0 \\
0\end{array}$ & $\begin{array}{l}124 \\
823\end{array}$ \\
\hline $\begin{array}{l}\text { air05 } \\
\text { airkio }\end{array}$ & $\begin{array}{l}7195 \\
138\end{array}$ & $\begin{array}{r}7195 \\
415\end{array}$ & 0 & $\begin{array}{r}426 \\
1048\end{array}$ \\
\hline $\begin{array}{l}\text { arkinol } \\
\text { bel13a }\end{array}$ & $\begin{array}{r}1388 \\
133\end{array}$ & $\begin{array}{r}415 \\
39\end{array}$ & $\begin{array}{r}1233 \\
32\end{array}$ & $\begin{array}{r}1048 \\
123\end{array}$ \\
\hline & & & & \\
\hline $\begin{array}{l}\text { Dendaz } \\
\text { cap6000 }\end{array}$ & 6000 & 6000 & 0 & 2174 \\
\hline $\begin{array}{l}\text { dano3mimp } \\
\text { danoint }\end{array}$ & $\begin{array}{r}13873 \\
521\end{array}$ & $\begin{array}{l}552 \\
56\end{array}$ & $\begin{array}{l}0 \\
0\end{array}$ & $\begin{array}{c}3202 \\
664\end{array}$ \\
\hline $\begin{array}{l}\text { demulti } \\
\text { dempin }\end{array}$ & 548 & 75 & 0 & 290 \\
\hline $\begin{array}{l}\text { dsbmip } \\
\text { egout }\end{array}$ & $\begin{array}{r}1877 \\
141\end{array}$ & $\begin{array}{r}160 \\
55\end{array}$ & $\begin{array}{l}0 \\
0\end{array}$ & $\begin{array}{r}1182 \\
98\end{array}$ \\
\hline $\begin{array}{l}\text { enigma } \\
\text { fiber }\end{array}$ & $\begin{array}{l}100 \\
1298\end{array}$ & $\begin{array}{l}100 \\
1254\end{array}$ & $\begin{array}{l}0 \\
0\end{array}$ & 363 \\
\hline fixnet6 & 878 & 378 & 0 & 478 \\
\hline $\begin{array}{l}\text { flugp1 } \\
\text { gen }\end{array}$ & $\begin{array}{l}18 \\
870\end{array}$ & $\begin{array}{r}0 \\
144\end{array}$ & $\begin{array}{r}11 \\
6\end{array}$ & $\begin{array}{r}18 \\
780\end{array}$ \\
\hline$\underset{\substack{\text { gesa22 } \\
\text { gesa2_o }}}{ }$ & ${ }_{1224}^{1224}$ & $\begin{array}{l}240 \\
384\end{array}$ & $\begin{array}{l}168 \\
336\end{array}$ & $\begin{array}{l}1392 \\
1248\end{array}$ \\
\hline gesa3 & 1152 & $\begin{array}{l}216 \\
336\end{array}$ & $\begin{array}{l}168 \\
336\end{array}$ & 1368 \\
\hline $\begin{array}{l}\text { gesa3_o } \\
\text { gt2 }\end{array}$ & 188 & 24 & $\begin{array}{l}300 \\
164\end{array}$ & $\begin{array}{r}1224 \\
29\end{array}$ \\
\hline $\begin{array}{l}\text { harp2 } \\
\text { harphos }\end{array}$ & 2993 & 2993 & 0 & 112 \\
\hline $\begin{array}{l}\text { Khb05250 } \\
11521 \mathrm{av}\end{array}$ & $\begin{array}{l}1350 \\
1989\end{array}$ & $\begin{array}{r}24 \\
1989\end{array}$ & 0 & 97 \\
\hline lseu & 89 & 89 & 0 & 28 \\
\hline $\begin{array}{l}\text { markshare1 } \\
\text { markshare } 2\end{array}$ & $\frac{62}{74}$ & $\begin{array}{l}50 \\
60\end{array}$ & $\begin{array}{l}0 \\
0\end{array}$ & $\frac{6}{7}$ \\
\hline mas74 & 151 & 150 & 0 & 13 \\
\hline $\begin{array}{l}\text { mas76 } \\
\text { misc03 }\end{array}$ & $\begin{array}{l}151 \\
160\end{array}$ & $\begin{array}{l}150 \\
159\end{array}$ & $\begin{array}{l}0 \\
0\end{array}$ & $\begin{array}{l}12 \\
96 \\
\end{array}$ \\
\hline $\begin{array}{l}\operatorname{misc06} \\
\text { misc07 }\end{array}$ & $\begin{aligned} 1808 \\
260\end{aligned}$ & $\begin{array}{l}112 \\
259\end{array}$ & $\begin{array}{l}0 \\
0\end{array}$ & $\begin{array}{l}820 \\
212\end{array}$ \\
\hline mitre & 10724 & 10724 & 0 & 2054 \\
\hline mkc & $\begin{array}{r}5325 \\
319\end{array}$ & $\begin{array}{r}5323 \\
319\end{array}$ & 0 & 3411 \\
\hline $\begin{array}{l}\text { mod008 } \\
\text { mod010 }\end{array}$ & 2655 & 2655 & 0 & $\begin{array}{r}6 \\
146\end{array}$ \\
\hline $\begin{array}{l}\text { modglob } \\
\text { noswot }\end{array}$ & $\begin{array}{l}422 \\
128\end{array}$ & $\begin{array}{l}98 \\
75\end{array}$ & 25 & $\begin{array}{l}291 \\
182\end{array}$ \\
\hline $\begin{array}{l}\text { Hos } \\
\text { nTw4 }\end{array}$ & 87482 & 87482 & 0 & 36 \\
\hline p0033 & & & & 15 \\
\hline
\end{tabular}

MIPLib3 2/2
\begin{tabular}{|l|rrrr|}
\hline Instance & $n$ & Bin. & Int. & $m$ \\
\hline p0201 & 201 & 201 & 0 & 133 \\
p0282 & 282 & 282 & 0 & 241 \\
p0548 & 548 & 548 & 0 & 176 \\
p2756 & 2756 & 2756 & 0 & 755 \\
pk1 & 86 & 55 & 0 & 45 \\
pp08aCuTs & 240 & 64 & 0 & 246 \\
pp08a & 240 & 64 & 0 & 136 \\
qnet1 & 1541 & 1288 & 129 & 503 \\
qnet1_o & 1541 & 1288 & 129 & 456 \\
rgn & 180 & 100 & 0 & 24 \\
rentacar & 9557 & 55 & 0 & 6803 \\
rout & 556 & 300 & 15 & 291 \\
set1 11. & 712 & 240 & 0 & 492 \\
seymour & 1372 & 1372 & 0 & 4944 \\
stein27 & 27 & 27 & 0 & 118 \\
stein45 & 45 & 45 & 0 & 331 \\
swath & 6805 & 6724 & 0 & 884 \\
vpm1 & 378 & 168 & 0 & 234 \\
vpm2 & 378 & 168 & 0 & 234 \\
\hline
\end{tabular}

\begin{tabular}{|c|c|c|c|c|}
\hline Instance & $n$ & Bin. & Int. & $m$ \\
\hline a1c1s1 & 3648 & 192 & 0 & 3312 \\
\hline af low30a & 842 & $\begin{array}{r}421 \\
1364\end{array}$ & 0 & $\begin{array}{r}479 \\
1442\end{array}$ \\
\hline atlanta-ip & 48738 & $\begin{array}{l}1364 \\
46667\end{array}$ & 106 & 21731 \\
\hline $\begin{array}{l}\text { disctom } \\
\text { glass4 }\end{array}$ & 10000 & $\begin{array}{r}10000 \\
302\end{array}$ & $\begin{array}{l}0 \\
0\end{array}$ & 399 \\
\hline $\begin{array}{l}\text { glass } \\
\text { liu }\end{array}$ & 1154 & 1087 & 0 & $\begin{array}{r}3178 \\
2178\end{array}$ \\
\hline $\begin{array}{l}\text { manna81 } \\
\text { momentum 1 }\end{array}$ & $\begin{array}{l}3321 \\
5174\end{array}$ & $\begin{array}{r}18 \\
2349\end{array}$ & 3303 & $\begin{array}{r}6480 \\
42680\end{array}$ \\
\hline $\begin{array}{l}\text { momentum2 } \\
\text { moment }\end{array}$ & $\begin{array}{r}31732 \\
13040\end{array}$ & $\begin{array}{l}1808 \\
0909\end{array}$ & 1 & 24237 \\
\hline $\begin{array}{l}\text { mzzv11 } \\
\text { mzzv42z }\end{array}$ & $\begin{array}{l}10240 \\
11717\end{array}$ & $\begin{array}{r}9989 \\
11482\end{array}$ & 235 & $\begin{array}{r}9499 \\
10460\end{array}$ \\
\hline $\begin{array}{l}\text { net12 } \\
\text { neta }\end{array}$ & $\begin{array}{l}14115 \\
14661\end{array}$ & 1603 & 0 & $\begin{array}{l}14021 \\
725\end{array}$ \\
\hline $\begin{array}{l}\text { nsrand-ipx } \\
\text { opt 1217 }\end{array}$ & $\begin{array}{l}6621 \\
769\end{array}$ & $\begin{array}{r}6620 \\
768\end{array}$ & $\begin{array}{l}0 \\
0\end{array}$ & $\begin{array}{r}735 \\
64\end{array}$ \\
\hline $\begin{array}{l}\text { protfold } \\
\text { protforen }\end{array}$ & 1835 & 1835 & 00 & 2112 \\
\hline $\begin{array}{l}\text { rollsoou } \\
\text { timtab1 }\end{array}$ & 397 & 64 & 107 & 171 \\
\hline $\begin{array}{l}\text { timtab2 } \\
\operatorname{tr} 12-30\end{array}$ & $\begin{array}{r}675 \\
1080\end{array}$ & $\begin{array}{l}113 \\
360\end{array}$ & $\begin{array}{r}181 \\
0\end{array}$ & $\begin{array}{l}294 \\
750\end{array}$ \\
\hline
\end{tabular}

Table 12 MILP instance statistics. 


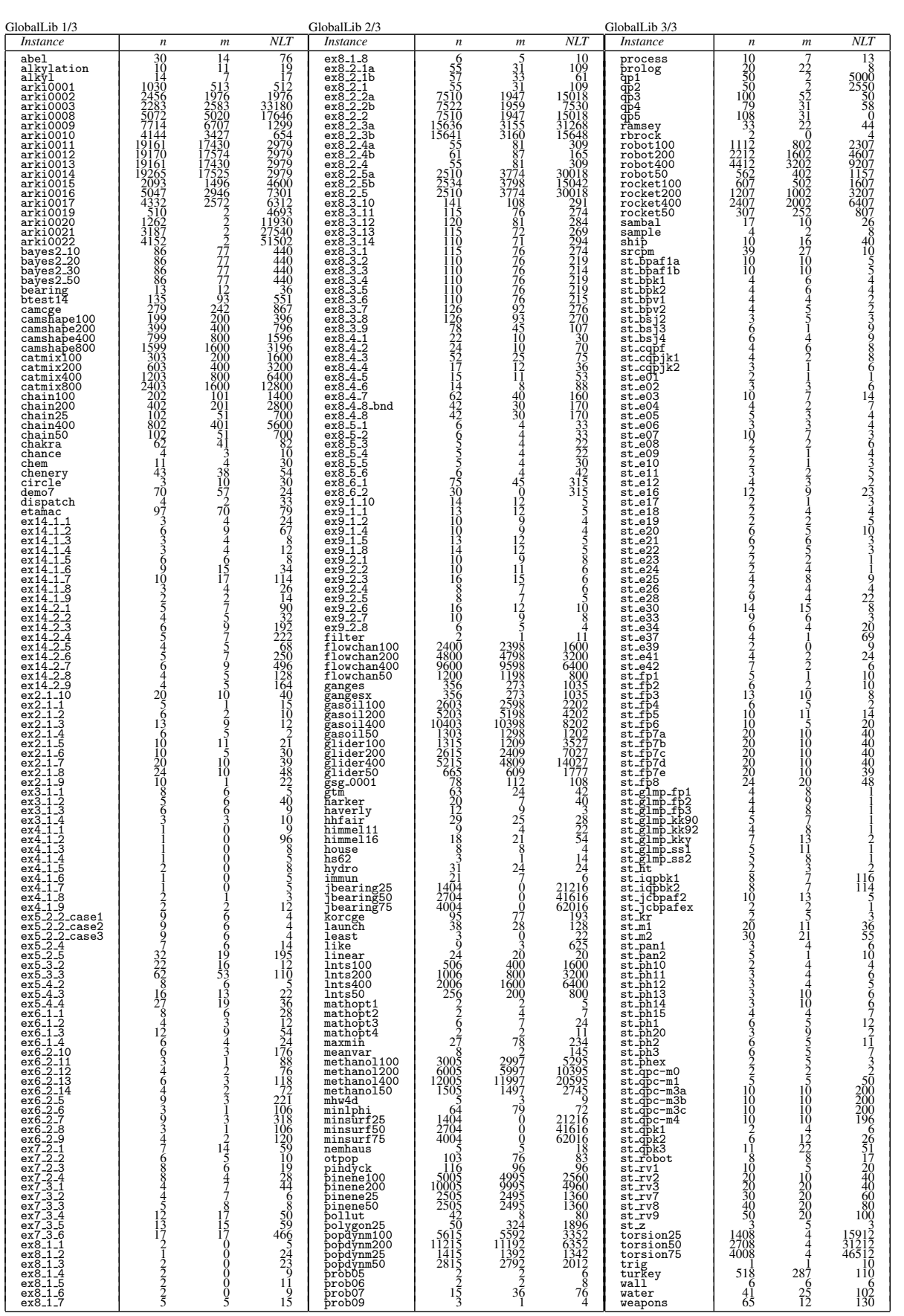

Table 13 NLP instance statistics. NLT is the number of nonlinear terms in the problem; AMPL errors on fct, worst. 


\begin{tabular}{|c|c|c|c|c|c|c|c|c|c|c|c|}
\hline IInstance 1 & $n$ & Bin. & Int. & $m$ & $N L T$ & 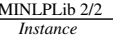 & & & & & \\
\hline 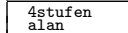 & 149 & $\begin{array}{l}48 \\
4\end{array}$ & $\begin{array}{l}0 \\
0\end{array}$ & 98 & 111 & $\begin{array}{l}n \text { nvs } \\
\text { nvs }\end{array}$ & 10 & 0 & 10 & & $\frac{5 L T}{50}$ \\
\hline $\begin{array}{l}\text { batchdes } \\
\text { batch }\end{array}$ & $\begin{array}{r}20 \\
46 \\
46\end{array}$ & $\begin{array}{r}9 \\
24 \\
\end{array}$ & $\begin{array}{l}0 \\
0 \\
0\end{array}$ & $\begin{array}{r}20 \\
.73\end{array}$ & $\begin{array}{r}13 \\
28 \\
28\end{array}$ & $\begin{array}{l}\text { nvs10 } \\
\text { nvs } 11 \\
\text { nvs } 12\end{array}$ & & $\begin{array}{l}0 \\
0 \\
0\end{array}$ & 2 & & $\begin{array}{l}16 \\
42 \\
02\end{array}$ \\
\hline $\begin{array}{l}\text { beuster } \\
\text { cecil1.13 }\end{array}$ & $\begin{array}{l}157 \\
800 \\
205\end{array}$ & $\begin{array}{r}52 \\
162 \\
87\end{array}$ & $\begin{array}{r}0 \\
18 \\
1\end{array}$ & $\begin{array}{l}114 \\
888 \\
884\end{array}$ & $\begin{array}{l}155 \\
\begin{array}{l}155 \\
799\end{array}\end{array}$ & 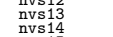 & & & & & $\begin{array}{l}158 \\
22\end{array}$ \\
\hline 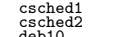 & $\begin{array}{l}717 \\
401 \\
402\end{array}$ & $\begin{array}{r}63 \\
308 \\
308\end{array}$ & $\begin{array}{r}0 \\
0 \\
0\end{array}$ & $\begin{array}{l}23 \\
138 \\
130\end{array}$ & $\begin{array}{l}16 \\
141 \\
040\end{array}$ & $\begin{array}{l}\text { nvs15 } \\
\text { nvs16 } \\
\text { nvs17 }\end{array}$ & & $\begin{array}{l}0 \\
0 \\
0\end{array}$ & $\begin{array}{l}3 \\
2 \\
7\end{array}$ & & $\begin{array}{r}9 \\
8 \\
392\end{array}$ \\
\hline $\begin{array}{l}\text { deb10 } \\
\text { debb } \\
\text { deb7 } \\
\text { debb }\end{array}$ & $\begin{array}{l}182 \\
4713 \\
837\end{array}$ & $\begin{array}{l}11 \\
20 \\
10\end{array}$ & $\begin{array}{l}11 \\
0 \\
10\end{array}$ & $\begin{array}{l}129 \\
507 \\
897\end{array}$ & $\begin{array}{l}390 \\
30108 \\
6644\end{array}$ & 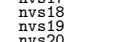 & $\begin{array}{r}6 \\
8 \\
16\end{array}$ & $\begin{array}{l}0 \\
0 \\
0\end{array}$ & $\begin{array}{l}6 \\
8 \\
5\end{array}$ & & $\begin{array}{l}558 \\
556 \\
122\end{array}$ \\
\hline $\begin{array}{l}\text { leb8 } \\
\text { debs } \\
\text { du-opt5 }\end{array}$ & $\begin{array}{l}823 \\
823 \\
200\end{array}$ & $\begin{array}{l}10 \\
10 \\
0\end{array}$ & $\begin{array}{l}10 \\
10 \\
13\end{array}$ & $\begin{array}{l}897 \\
917\end{array}$ & $\begin{array}{l}6144 \\
66144 \\
2326\end{array}$ & $\begin{array}{l}\text { nvs } \\
\text { nvs } 21 \\
\text { nss22 }\end{array}$ & 3 & $\begin{array}{l}0 \\
0 \\
0\end{array}$ & $\frac{2}{4}$ & & $\begin{array}{l}121 \\
48 \\
48\end{array}$ \\
\hline $\begin{array}{l}\text { du-opt } \\
\text { dulf } \\
\text { elf } \\
\text { eniplac }\end{array}$ & $\begin{array}{l}20 \\
144 \\
140\end{array}$ & $\begin{array}{l}20 \\
24 \\
24\end{array}$ & $\begin{aligned} 13 \\
0 \\
0\end{aligned}$ & $\begin{array}{r}99 \\
38 \\
189\end{array}$ & $\begin{aligned} 2336 \\
236 \\
27 \\
90\end{aligned}$ & $\begin{array}{l}\text { nus } 23 \\
\text { nvs } 24 \\
\text { of } 22\end{array}$ & $\begin{array}{r}99 \\
114 \\
114\end{array}$ & $\begin{array}{r}0 \\
0 \\
42\end{array}$ & $\begin{array}{c}9 \\
10 \\
0\end{array}$ & $\begin{array}{r}99 \\
211 \\
211\end{array}$ & $\begin{array}{l}762 \\
1020 \\
14\end{array}$ \\
\hline 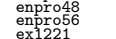 & $\begin{array}{l}154 \\
128 \\
5\end{array}$ & $\begin{array}{l}92 \\
73 \\
3\end{array}$ & $\begin{array}{l}0 \\
0 \\
0\end{array}$ & $\begin{array}{l}215 \\
192 \\
5\end{array}$ & $\begin{array}{l}35 \\
31 \\
2\end{array}$ & $\begin{array}{l}\text { ofer } \\
\text { oarer } \\
\text { oil12 }\end{array}$ & $\begin{array}{r}114 \\
9397\end{array}$ & $\begin{array}{r}42 \\
3 \\
2\end{array}$ & $\begin{array}{l}0 \\
0 \\
0\end{array}$ & $\begin{array}{r}211 \\
927\end{array}$ & $\begin{array}{r}14 \\
1981 \\
1981\end{array}$ \\
\hline 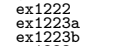 & 7 & & $\begin{array}{l}0 \\
0 \\
0\end{array}$ & 3 & $\begin{array}{r}3 \\
3 \\
17\end{array}$ & $\begin{array}{l}\text { o1 } \\
\text { ortez } \\
\text { parallel } \\
\text { probo }\end{array}$ & $\begin{array}{r}1535 \\
805 \\
205\end{array}$ & $\begin{array}{l}19 \\
18 \\
25\end{array}$ & $\begin{array}{l}0 \\
0 \\
0\end{array}$ & $\begin{array}{r}1546 \\
115 \\
115\end{array}$ & $\begin{array}{r}2811 \\
570 \\
280\end{array}$ \\
\hline $\begin{array}{l}\text { ex1223 } \\
\text { ex 1224 } \\
\text { ex 1225 }\end{array}$ & $\begin{array}{l}11 \\
11 \\
8\end{array}$ & & 0 & $\begin{array}{c}13 \\
17 \\
10\end{array}$ & i7 & $\begin{array}{l}\text { prob02 } \\
\text { probo3 } \\
\text { prob10 }\end{array}$ & $\begin{array}{l}6 \\
\frac{2}{3} \\
3\end{array}$ & $\begin{array}{l}0 \\
0 \\
0\end{array}$ & $\begin{array}{l}6 \\
1 \\
1\end{array}$ & & $\frac{9}{9}$ \\
\hline 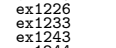 & $\begin{array}{l}55 \\
52 \\
68\end{array}$ & $\begin{array}{r}3 \\
12 \\
16\end{array}$ & $\begin{array}{l}0 \\
0 \\
0 \\
0\end{array}$ & $\begin{array}{l}65 \\
64 \\
96\end{array}$ & $\begin{array}{l}68 \\
68 \\
32\end{array}$ & $\begin{array}{l}\text { procsel } \\
\text { product2 } \\
\text { product }\end{array}$ & $\begin{array}{l}280 \\
1553 \\
1553\end{array}$ & $\begin{array}{l}128 \\
107\end{array}$ & $\begin{array}{l}0 \\
0 \\
0\end{array}$ & $\begin{array}{l}3125 \\
1925\end{array}$ & $\begin{array}{l}2112 \\
528 \\
528\end{array}$ \\
\hline 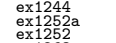 & $\begin{array}{l}95 \\
24 \\
39\end{array}$ & $\begin{aligned} 23 \\
15 \\
15\end{aligned}$ & $\begin{array}{l}0 \\
6 \\
0\end{array}$ & $\begin{array}{l}129 \\
34 \\
43\end{array}$ & $\begin{array}{l}46 \\
75 \\
75\end{array}$ & $\begin{array}{l}\text { pump } \\
\text { qappu } \\
\text { ravem }\end{array}$ & 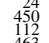 & $\begin{array}{r}205 \\
253 \\
53\end{array}$ & $\begin{array}{l}0 \\
0 \\
1 \\
1\end{array}$ & $\begin{array}{l}234 \\
255 \\
186\end{array}$ & $\begin{array}{l}225 \\
43\end{array}$ \\
\hline 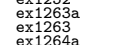 & $\begin{array}{l}24 \\
92 \\
24\end{array}$ & $\begin{array}{r}4 \\
72 \\
4\end{array}$ & $\begin{array}{r}20 \\
0 \\
20\end{array}$ & $\begin{array}{l}\frac{13}{35} \\
55\end{array}$ & $\begin{array}{l}16 \\
16 \\
16\end{array}$ & $\begin{array}{l}\text { riskab } \\
\text { saa } 2 \text { - } \\
\text { sepp } 1\end{array}$ & $\begin{array}{r}463 \\
4407 \\
29\end{array}$ & $\begin{array}{r}12 \\
400 \\
2\end{array}$ & $\begin{array}{l}2 \\
0 \\
0\end{array}$ & $\begin{array}{l}580 \\
6205 \\
31\end{array}$ & $14369^{6}$ \\
\hline 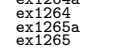 & $\begin{array}{l}8.5 \\
835 \\
130 \\
130\end{array}$ & $\begin{array}{r}68 \\
150\end{array}$ & $\begin{array}{r}0 \\
30 \\
0\end{array}$ & $\begin{array}{l}55 \\
54 \\
74\end{array}$ & $\begin{array}{l}16 \\
25 \\
25\end{array}$ & $\begin{array}{l}\text { spacees5a } \\
\text { spacens } \\
\text { space960 }\end{array}$ & $\begin{array}{r}383 \\
5833 \\
5577\end{array}$ & $\begin{array}{l}240 \\
750 \\
30\end{array}$ & $\begin{array}{r}0 \\
0 \\
960\end{array}$ & $\begin{array}{r}201 \\
203 \\
6497\end{array}$ & $\begin{aligned} 101 \\
101 \\
3748\end{aligned}$ \\
\hline 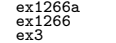 & $\begin{array}{l}48 \\
180 \\
32\end{array}$ & $\begin{array}{r}138 \\
8\end{array}$ & $\begin{array}{r}42 \\
0 \\
0\end{array}$ & $\begin{array}{l}53 \\
51 \\
31\end{array}$ & $\begin{array}{l}36 \\
36 \\
7\end{array}$ & $\begin{array}{l}\text { spectraz } \\
\text { sting } \\
\text { st-ents } \\
\text { s.e-14 }\end{array}$ & $\begin{array}{l}10 \\
17 \\
\end{array}$ & & $\begin{array}{l}0 \\
0 \\
0\end{array}$ & $\begin{array}{r}13 \\
2 \\
2\end{array}$ & $\begin{array}{r}r 04 \\
19 \\
1\end{array}$ \\
\hline & $\begin{array}{l}37 \\
22 \\
66\end{array}$ & $\begin{array}{r}25 \\
6 \\
12\end{array}$ & $\begin{array}{l}0 \\
0 \\
0\end{array}$ & 31 & $\begin{array}{r}254 \\
6 \\
6\end{array}$ & $\begin{array}{l}\text { ste-e14 } \\
\text { ste-e15 } \\
\text { st-e27 }\end{array}$ & $\begin{array}{l}11 \\
5\end{array}$ & & $\begin{array}{l}0 \\
0 \\
0\end{array}$ & $\begin{array}{r}13 \\
5 \\
6\end{array}$ & 17 \\
\hline $\begin{array}{l}\text { face } \\
\text { faedrayy } \\
\text { feedtray }\end{array}$ & $\begin{array}{l}66 \\
88 \\
98\end{array}$ & $\begin{array}{l}12 \\
36 \\
7\end{array}$ & $\begin{array}{l}0 \\
0 \\
0\end{array}$ & $\begin{array}{r}33 \\
284 \\
92\end{array}$ & $\begin{array}{l}865 \\
977\end{array}$ & $\begin{array}{l}\text { ste-e29 } \\
\text { st-e31 } \\
\text { st-e32 } \\
\text { ste } 25\end{array}$ & $\begin{array}{l}111 \\
112 \\
32\end{array}$ & $\begin{array}{r}24 \\
0 \\
0\end{array}$ & $\begin{array}{r}0 \\
0 \\
19\end{array}$ & $\begin{array}{l}135 \\
13 \\
38\end{array}$ & 92 \\
\hline 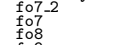 & $\begin{array}{l}114 \\
114 \\
146\end{array}$ & $\begin{array}{l}42 \\
42 \\
56\end{array}$ & $\begin{array}{l}0 \\
0 \\
0\end{array}$ & $\begin{array}{l}211 \\
211 \\
273\end{array}$ & $\begin{array}{l}14 \\
16 \\
16\end{array}$ & $\begin{array}{l}\text { ste-e35 } \\
\text { ste-e36 } \\
\text { st-e38 }\end{array}$ & $\begin{array}{r}32 \\
2 \\
4\end{array}$ & $\begin{array}{l}1 \\
0 \\
0 \\
0\end{array}$ & $\begin{array}{l}0 \\
1 \\
0 \\
3\end{array}$ & $\begin{array}{r}39 \\
2 \\
3 \\
3\end{array}$ & $\begin{array}{l}36 \\
17 \\
17\end{array}$ \\
\hline $\begin{array}{l}\text { fog } \\
\text { fuel } \\
\text { fuzzy }\end{array}$ & $\begin{array}{l}182 \\
15 \\
896\end{array}$ & $\begin{array}{r}72 \\
320 \\
120\end{array}$ & $\begin{array}{l}0 \\
0 \\
0\end{array}$ & $\begin{array}{l}343 \\
315 \\
1056\end{array}$ & $\begin{array}{l}18 \\
12 \\
72\end{array}$ & 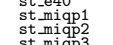 & ${ }^{4}$ & 0 & $\begin{array}{l}3 \\
0 \\
2\end{array}$ & & $\begin{array}{r}10 \\
10 \\
4\end{array}$ \\
\hline $\begin{array}{l}\text { gasnet } \\
\text { gastrans } \\
\text { gotrats }\end{array}$ & $\begin{array}{l}90 \\
106\end{array}$ & $\begin{array}{l}10 \\
21 \\
21\end{array}$ & 0 & $\begin{array}{l}69 \\
149 \\
4\end{array}$ & $\begin{array}{l}163 \\
45\end{array}$ & 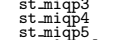 & $\begin{array}{l}2 \\
7 \\
7\end{array}$ & $\begin{array}{l}0 \\
3 \\
2\end{array}$ & $\begin{array}{l}2 \\
0 \\
0\end{array}$ & $1 \frac{13}{3}$ & $\begin{array}{l}2 \\
6 \\
4\end{array}$ \\
\hline $\begin{array}{l}\text { 然bdar2 } \\
\text { gear3 } \\
\text { geart }\end{array}$ & $\begin{array}{r}4 \\
28 \\
\end{array}$ & $\begin{array}{r}2^{3} \\
0 \\
0\end{array}$ & $\begin{array}{l}0 \\
0\end{array}$ & & $\begin{array}{l}4 \\
4 \\
4\end{array}$ & 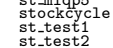 & $\begin{array}{r}480 \\
5 \\
6\end{array}$ & $\begin{array}{r}43 \frac{2}{5} \\
5\end{array}$ & $\begin{array}{l}0 \\
0 \\
0 \\
1\end{array}$ & $\begin{array}{r}97 \\
1 \\
2\end{array}$ & $\begin{array}{l}8 \\
10\end{array}$ \\
\hline $\begin{array}{l}\text { gear } \\
\text { gear } \\
\text { Ekocis }\end{array}$ & 4 & $\begin{array}{r}0 \\
3 \\
3\end{array}$ & $\begin{array}{l}0 \\
0\end{array}$ & 0 & $\begin{array}{r}4 \\
4 \\
3\end{array}$ & $\begin{array}{l}\text { stt-test3 } \\
\text { st-test4t } \\
\text { st-test5 }\end{array}$ & $\begin{array}{l}13 \\
10 \\
10\end{array}$ & $\begin{array}{l}10 \\
2 \\
10\end{array}$ & $\begin{array}{l}3 \\
4 \\
0\end{array}$ & $\begin{array}{r}10 \\
5 \\
11\end{array}$ & $\begin{array}{l}10 \\
14 \\
14\end{array}$ \\
\hline 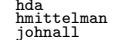 & $\begin{array}{l}713 \\
196 \\
194\end{array}$ & $\begin{array}{l}13 \\
15 \\
188\end{array}$ & $\begin{array}{r}0 \\
0 \\
0\end{array}$ & 719 & $\begin{array}{r}480 \\
238 \\
17860\end{array}$ & 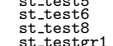 & $\begin{array}{l}10 \\
24 \\
10\end{array}$ & $\begin{array}{r}10 \\
0 \\
0\end{array}$ & $\begin{array}{r}0 \\
24 \\
10\end{array}$ & $\begin{array}{r}11 \\
20 \\
20\end{array}$ & $\begin{array}{l}20 \\
20 \\
48\end{array}$ \\
\hline $\begin{array}{l}\text { lomplicx } \\
\text { ma } \\
\text { me }\end{array}$ & $\begin{array}{l}986 \\
866 \\
866\end{array}$ & $\begin{array}{l}67 \\
30 \\
30\end{array}$ & $\begin{array}{r}785 \\
0 \\
0\end{array}$ & $\begin{array}{r}87 \\
437 \\
157\end{array}$ & $\begin{array}{r}556 \\
12 \\
12\end{array}$ & 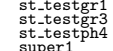 & $\begin{array}{r}10 \\
20 \\
3 \\
1308\end{array}$ & $\begin{array}{r}0 \\
0 \\
0 \\
4\end{array}$ & $\begin{aligned} 10 \\
20 \\
3 \\
0\end{aligned}$ & $\begin{array}{r}25 \\
10 \\
169 \\
169\end{array}$ & $\begin{array}{r}20 \\
40 \\
254 \\
254\end{array}$ \\
\hline $\begin{array}{l}\text { IIf } \\
\text { meanvarx } \\
\text { mounsar } \\
\text { nousi }\end{array}$ & 114 & $\begin{array}{l}42 \\
42 \\
14\end{array}$ & $\begin{array}{l}0 \\
0 \\
0\end{array}$ & $\begin{array}{l}211 \\
211\end{array}$ & $\begin{array}{l}14 \\
156 \\
66\end{array}$ & $\begin{array}{l}\text { Super1 } \\
\text { super2 } \\
\text { super3 }\end{array}$ & 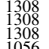 & $\begin{array}{l}444 \\
44 \\
44\end{array}$ & $\begin{array}{l}0 \\
0 \\
0 \\
0\end{array}$ & $\begin{array}{l}1659 \\
1659 \\
1659 \\
1342\end{array}$ & $\begin{array}{l}2545 \\
2545 \\
2545 \\
250\end{array}$ \\
\hline 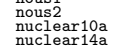 & $\begin{array}{r}500 \\
13010 \\
992\end{array}$ & $\begin{array}{r}0 \\
10920 \\
600\end{array}$ & $\begin{array}{l}0 \\
0 \\
0\end{array}$ & $\begin{array}{r}43 \\
3393 \\
633\end{array}$ & $\begin{array}{r}66 \\
31988 \\
3984\end{array}$ & $\begin{array}{l}\text { superert } \\
\text { synheat } \\
\text { synthes } 1\end{array}$ & $\begin{array}{r}1056 \\
56 \\
6\end{array}$ & $\begin{array}{l}44 \\
12 \\
\frac{2}{3} \\
3\end{array}$ & $\begin{array}{l}0 \\
0 \\
0 \\
0\end{array}$ & $\begin{array}{r}1343 \\
64 \\
6\end{array}$ & $\begin{array}{r}r 169 \\
82 \\
11 \\
9\end{array}$ \\
\hline 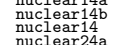 & $\begin{array}{l}1568 \\
1562 \\
992\end{array}$ & $\begin{array}{l}600 \\
576 \\
600\end{array}$ & $\begin{array}{l}0 \\
0 \\
0\end{array}$ & $\begin{array}{l}1725 \\
1226 \\
633\end{array}$ & $\begin{array}{l}3408 \\
4272 \\
3984\end{array}$ & $\begin{array}{l}\text { synthese } 2 \\
\text { synthes } \\
\text { tIn12 } 3\end{array}$ & $\begin{array}{r}17 \\
168 \\
168\end{array}$ & $\begin{array}{r}5 \\
12 \\
12\end{array}$ & $\begin{array}{r}0 \\
156 \\
156\end{array}$ & $\begin{array}{l}14 \\
72 \\
72\end{array}$ & $\begin{array}{r}9 \\
144 \\
144\end{array}$ \\
\hline 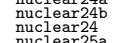 & $\begin{array}{l}1568 \\
1562 \\
1502\end{array}$ & $\begin{array}{l}600 \\
576 \\
560\end{array}$ & $\begin{array}{l}0 \\
0 \\
0 \\
0\end{array}$ & 1793 & $\begin{array}{l}3408 \\
4272 \\
4215\end{array}$ & $\begin{array}{l}\operatorname{tin} 2 \\
\operatorname{tin} 4 \\
\operatorname{tin} 5\end{array}$ & $\begin{array}{l}8 \\
24 \\
35\end{array}$ & $\begin{array}{l}2 \\
\frac{2}{5}\end{array}$ & $\begin{array}{l}60 \\
30 \\
30\end{array}$ & $\begin{array}{l}12 \\
24 \\
30\end{array}$ & $\begin{array}{l}4 \\
16 \\
25\end{array}$ \\
\hline 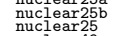 & 16678 & $\begin{array}{l}650 \\
625 \\
625\end{array}$ & $\begin{array}{l}0 \\
0 \\
0\end{array}$ & 1909 & $\begin{array}{l}3590 \\
3590 \\
45900\end{array}$ & $\begin{array}{l}\operatorname{tin} 6 \\
\operatorname{tnn} 7 \\
\operatorname{tn} 89\end{array}$ & $\begin{array}{l}48 \\
63 \\
63 \\
48\end{array}$ & 6 & $\begin{array}{l}42 \\
56\end{array}$ & $\begin{array}{l}36 \\
42 \\
53\end{array}$ & $\begin{array}{l}36 \\
49 \\
36\end{array}$ \\
\hline 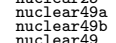 & $\begin{array}{l}x_{3341} \\
5742 \\
5735\end{array}$ & $\begin{array}{r}2450 \\
2450 \\
2400\end{array}$ & $\begin{array}{l}0 \\
0 \\
0 \\
0\end{array}$ & $\begin{array}{l}1431 \\
62233 \\
3873\end{array}$ & $\begin{array}{l}10826 \\
8825 \\
10541\end{array}$ & $\begin{array}{l}\text { t1oss } \\
\text { t11 1112 } \\
\text { t112 } 2\end{array}$ & $\begin{array}{l}48 \\
832 \\
37\end{array}$ & $\begin{array}{l}656 \\
635 \\
31\end{array}$ & $\begin{array}{l}42 \\
\frac{42}{2} \\
2\end{array}$ & $\begin{array}{r}53 \\
384 \\
24\end{array}$ & $\begin{array}{r}36 \\
288 \\
8\end{array}$ \\
\hline $\begin{array}{l}\text { nnclearvat } \\
\text { nuclearvervo }\end{array}$ & 351 & $\begin{array}{l}168 \\
1.68 \\
1.88 \\
\end{array}$ & $\begin{array}{l}0 \\
0 \\
0\end{array}$ & $\begin{array}{l}31 \\
31 \\
31\end{array}$ & $\begin{array}{l}1664 \\
1628 \\
1628\end{array}$ & 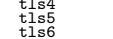 & $\begin{array}{l}115 \\
161 \\
221\end{array}$ & $\begin{array}{l}855 \\
131 \\
133\end{array}$ & $\begin{array}{l}4 \\
5 \\
6\end{array}$ & $\begin{array}{l}64 \\
90 \\
100\end{array}$ & $\begin{array}{l}32 \\
32 \\
72\end{array}$ \\
\hline $\begin{array}{l}\text { nuclearve } \\
\text { nuclearve } \\
\text { nuclearve }\end{array}$ & $\begin{array}{l}351 \\
351 \\
351\end{array}$ & $\begin{array}{l}168 \\
168 \\
168 \\
\end{array}$ & $\begin{array}{l}0 \\
0 \\
0\end{array}$ & $\begin{array}{l}317 \\
317 \\
317\end{array}$ & $\begin{array}{l}1258 \\
2504 \\
2504\end{array}$ & $\begin{array}{l}\text { tist } \\
\text { t1tr }\end{array}$ & $\begin{array}{r}345 \\
48 \\
48\end{array}$ & $\begin{array}{l}289 \\
212\end{array}$ & $\begin{array}{r}7 \\
36 \\
36\end{array}$ & $\begin{array}{r}154 \\
154\end{array}$ & $\begin{array}{r}98 \\
27 \\
0.72\end{array}$ \\
\hline $\begin{array}{l}\text { nuctearvf } \\
\text { nvsolor }\end{array}$ & 35 & $\begin{array}{c}168 \\
160 \\
0\end{array}$ & & & $\begin{array}{l}2504 \\
13 \\
13\end{array}$ & 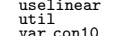 & $\begin{array}{l}6792 \\
6146 \\
53\end{array}$ & $\begin{array}{r}58 \\
28 \\
10\end{array}$ & $\begin{array}{l}0 \\
0 \\
2\end{array}$ & $\begin{array}{l}7030 \\
168 \\
464\end{array}$ & $\begin{array}{l}9549 \\
4368 \\
436\end{array}$ \\
\hline $\begin{array}{l}\text { nvs02 } \\
\text { nvso3 } \\
\text { nvos } 04\end{array}$ & & $\begin{array}{l}0 \\
0\end{array}$ & & ${ }_{0}^{2}$ & $\begin{array}{r}23 \\
4 \\
4\end{array}$ & 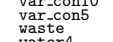 & $\begin{array}{r}5173 \\
2484\end{array}$ & $\begin{array}{l}100 \\
400\end{array}$ & $\frac{2}{0}$ & $\begin{array}{l}4664 \\
1991 \\
1912\end{array}$ & $\begin{array}{l}4368 \\
1368 \\
1368\end{array}$ \\
\hline 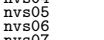 & & $\begin{array}{l}0 \\
0 \\
0\end{array}$ & & 9 & $\begin{array}{l}48 \\
13 \\
13\end{array}$ & 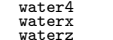 & $\begin{array}{l}195 \\
195 \\
195\end{array}$ & $\begin{array}{l}126 \\
14 \\
16\end{array}$ & $\begin{array}{l}0 \\
0 \\
0\end{array}$ & $\begin{array}{l}137 \\
134 \\
137\end{array}$ & $\begin{array}{l}32 \\
102 \\
32\end{array}$ \\
\hline & & & 2 & & ${ }_{11}^{4}$ & $\begin{array}{l}\text { waterz } \\
\text { windfac }\end{array}$ & $\begin{array}{l}199 \\
14 \\
\end{array}$ & $\begin{array}{r}126 \\
0\end{array}$ & $\begin{array}{l}0 \\
3 \\
\end{array}$ & & \\
\hline
\end{tabular}

Table 14 MINLP instance statistics. NLT is the number of nonlinear terms in the problem. AMPL errors on blendgap, meanvarxsc, water3, waterful2, watersbp, waters, watersym1, watersym2 (MINLPLib). 